\title{
Advection\#dominated Accretion Model of the Black Hole V404 Cygni in Quiescence
}

\section{Citation}

Narayan, Ramesh, Didier Barret, and Jeffrey E. McClintock. 1997. "Advection\#dominated Accretion Model of the Black Hole V404 Cygni in Quiescence." The Astrophysical Journal 482 (1): 448-64. https://doi.org/10.1086/304134.

\section{Permanent link}

http://nrs.harvard.edu/urn-3:HUL.InstRepos:41384908

\section{Terms of Use}

This article was downloaded from Harvard University's DASH repository, and is made available under the terms and conditions applicable to Other Posted Material, as set forth at http:// nrs.harvard.edu/urn-3:HUL.InstRepos:dash.current.terms-of-use\#LAA

\section{Share Your Story}

The Harvard community has made this article openly available.

Please share how this access benefits you. Submit a story.

Accessibility 


\title{
Advection-Dominated Accretion Model of the Black Hole V404 Cyg in Quiescence
}

\author{
Ramesh Narayan, Didier Barret, Jeffrey E. McClintock \\ Harvard-Smithsonian Center for Astrophysics, 60 Garden St., Cambridge, MA 02138; \\ rnarayan@cfa.harvard.edu, barret@cesr.cnes.fr, jmcclintock@cfa.harvard.edu
}

\begin{abstract}
We have analyzed archival ASCA data on the soft X-ray transient source V404 Cyg in quiescence. We find that in the energy range 0.7 to $8.5 \mathrm{keV}$ the spectrum is a hard power-law with a photon spectral index between 1.8 and 2.6 (90\% confidence limits). We present a model of V404 Cyg in which the accretion flow has two components: (1) an outer thin disk with a small annular extent, and (2) a large interior region where the flow is advection-dominated. Nearly all the radiation in the infrared, optical, UV and X-ray bands is from the advection-dominated zone; the thin disk radiates primarily in the infrared where it contributes about ten percent of the observed flux. The spectrum we calculate with this model is in excellent agreement with the ASCA X-ray data presented here, as well as with previous optical data. Moreover, the fit is very insensitive to the choice of parameters such as black hole mass, orbital inclination, viscosity coefficient $\alpha$, and magnetic field strength. We consider the success of the model to be strong support for the advection-dominated accretion paradigm, and further evidence of the black hole nature of V404 Cyg. We discuss strategies whereby systems with advection-dominated accretion could be used to prove the reality of event horizons in black holes.
\end{abstract}

Subject headings: accretion: accretion disks — binaries: close — black hole physics - X-ray binaries

\section{Introduction.}

Soft X-ray transients (SXTs) are among the best-known examples of accreting black holes (van Paradijs \& McClintock 1995, Tanaka \& Shibazaki 1996). Several of these X-ray binaries have measured mass functions larger than $3 M_{\odot}$, which exceeds the maximum mass of a neutron star, thereby confirming the black hole nature of the systems. Since 
SXTs are among the most accessible black holes for observational study, much effort has gone into observing and modeling them.

SXTs occasionally have outbursts lasting several months in which they flare up to a luminosity comparable to the Eddington limit. Most of the time, however, these sources are in a quiescent state with a luminosity several orders of magnitude below Eddington.

Observations of quiescent systems are difficult, but have nevertheless been carried out recently for a few systems in the optical, UV and X-ray bands. These observations have revealed a serious problem for models of quiescent SXTs. Specifically, in at least one system, namely A0620-00 (McClintock, Horne \& Remillard 1995), the optical and $\mathrm{X}$-ray data are inconsistent with any accretion model based purely on a thin accretion disk. Narayan, McClintock \& Yi (1996, hereafter NMY) showed that the optical data in A0620-00 can be fitted with a thin disk only if the inner edge of the disk is at several thousand Schwarzschild radii; however, such a model cannot explain the X-ray flux. On the other hand, if one tries to fit the X-ray data with a thin disk extending down to the black hole, the spectral shape does not fit the observations, and the model disagrees strongly with the optical, UV and EUV constraints.

The outbursts of SXTs are usually explained by means of a thermal limit cycle model (Mineshige \& Wheeler 1989). In this model, the entire accretion flow, from the outer edge down to the last stable orbit at three Schwarzschild radii, is assumed to be in the form of a thin disk, both in quiescence and in outburst. The fact that the observed spectrum in quiescence is inconsistent with a pure thin disk is clearly a problem for this model. Furthermore, the estimated recurrence time between outbursts disagrees seriously with observational constraints (Lasota, Narayan \& Yi 1996b).

These difficulties were overcome in a new model of quiescent SXTs proposed by NMY. In this model, the accretion flow occurs as a thin disk only outside a transition radius $r_{\text {tr }}$ (we use $r$ to represent the dimensionless radius in Schwarzschild units), while for the energetically important region $r<r_{\text {tr }}$ the accretion is via an advection-dominated accretion flow (ADAF). This model is able to explain the observed spectrum of A0620-00 (NMY), and at the same time appears also to predict a consistent recurrence time between outbursts (Lasota et al. 1996b). In the model of A0620-00 presented by NMY, $r_{\text {tr }} \sim$ few $\times 10^{3}$. However, in the models of V404 Cyg presented in this paper, $r_{\text {tr }}>10^{4}$. These models are thus dominated by the ADAF, and the thin disk plays only a minor role.

ADAFs are accretion flows in which most of the energy released via viscous dissipation remains in the accreting gas rather than being radiated away. They get their name from 
the fact that the bulk of the energy is advected with the flow. This is in contrast to a thin accretion disk, where essentially all the dissipated energy is radiated locally. ADAFs can occur in two distinct regimes.

At sufficiently high mass accretion rates $\dot{M}$, radiation is trapped by the accreting gas and is dragged into the central star (Katz 1977, Begelman 1978). In a comprehensive analysis, Abramowicz et al. (1988) showed that this regime of ADAFs corresponds to a fundamentally new branch of accretion. The branch has, however, not yet found application to real sources.

At low $\dot{M}$, a second branch of ADAFs is possible as the viscously heated gas becomes extremely optically thin and is unable to cool within the accretion time scale of the flow. The critical bottleneck to the cooling is the fact that the gas becomes a two-temperature plasma (Shapiro, Lightman \& Eardley 1976) so that energy transfer from ions, which acquire most of the viscously dissipated energy, to electrons is inefficient. Most of the energy then remains in the gas as thermal energy of the ions and is advected into the central star rather than being radiated. This regime of ADAFs was briefly discussed by Rees et al. (1982) when they considered an "ion torus" model in which they merged the two-temperature accretion model of Shapiro et al. (1976) with some new ideas on accretion tori and funnels (Fishbone \& Moncrief 1976, Abramowicz, Jaroszyński \& Sikora 1978). In this model the two-temperature gas forms a torus with open funnels through which jets are formed via the Blandford \& Znajek (1977) mechanism.

Recent theoretical work by a number of authors (Narayan \& Yi 1994, 1995ab, Abramowicz et al. 1995, Chen 1995, Chen et al. 1995, Narayan, Kato \& Honma 1997, Chen, Abramowicz \& Lasota 1997, see Narayan 1996a, 1997 for reviews) has led to a clear recognition of the low $\dot{M}$, optically thin regime of ADAFs as a new and distinct branch of accretion with dynamically consistent solutions. A number of the basic dynamical properties of optically thin ADAFs have been worked out, and it has been shown that these flows produce X-ray spectra similar to those observed in many accreting black holes. Furthermore, it is now clear that the low $\dot{M}$, optically thin ADAF branch is quite distinct from the Shapiro et al. (1976) solution. The distinction is important since the Shapiro et al. flow is known to be violently unstable (Piran 1978). The new ADAF branch, in contrast, is either fully stable (Abramowicz et al. 1995, Narayan \& Yi 1995b) or at most has a weak instability which is no threat to the global viability of the flow (Kato, Abramowicz \& Chen 1996).

Optically thin ADAFs appear to be perfect for explaining a variety of observations of accreting black holes, and there have indeed been several applications to real systems (Narayan, Yi \& Mahadevan 1995, NMY, Lasota et al. 1996a, Fabian \& Rees 1995, 
Mahadevan 1996). In particular, the NMY model of quiescent SXTs makes use of this solution to model the region of the accretion flow inside $r=r_{\text {tr }}$. The present paper describes further work on the NMY model, and is driven by three recent developments.

First, we have improved the modeling techniques significantly, both in the description of gas dynamics and in the treatment of radiation processes. Whereas the NMY work was based on a self-similar solution of the gas flow (Spruit et al. 1987, Narayan \& Yi 1994), we now make use of self-consistent global solutions with proper boundary conditions (Narayan et al. 1997, Chen et al. 1997). We have also improved the treatment of Comptonization. The details are described in $\S 2$ below.

Second, we focus on a prediction made by NMY, namely that the SXT source V404 Cyg in quiescence should have quite a hard X-ray spectrum, with a photon index of the order of 2. At the time of that paper, the data were not good enough to check the prediction. There was an indication based on ROSAT data that the spectrum may be extremely soft with $\alpha \sim 7$ (Wagner et al. 1994). However, a reanalysis of the same data by NMY gave $\alpha=4.0_{-1.5}^{+1.9}$. NMY suggested that an observation with the ASCA satellite would provide a definitive test of the model. V404 Cyg has now been observed by ASCA and the data are of sufficient quality for this test. We describe in $\S \S 3.1,3.2$ our analysis of the ASCA X-ray data and observations in other wavelength bands, and show in $\S 3.3$ that the ADAF model is in very good agreement with the observations. V404 Cyg is the most robust black hole candidate among the SXTs, since it has the largest mass function. It also has the strongest X-ray flux in quiescence. For both reasons, it is the best SXT to pursue for detailed testing of the ADAF paradigm. For completeness, we present in $\S 3.4$ a reanalysis of A0620-00 using the improved techniques of this paper.

Finally, Wheeler (1996) has recently argued that the original NMY model is inconsistent since the $\dot{M}$ of those models corresponds to a thermal instability in the outer thin disk. We show in $\S 4$ that the new models presented in this paper do not suffer from

the instability and are fully consistent. We also discuss in this section future opportunities for further testing the ADAF paradigm, and for establishing the reality of the event horizon in black holes.

\section{Modeling Techniques}

\subsection{The Model and its Parameters}

We consider a black hole of mass $M$ accreting gas at a steady mass accretion rate $\dot{M}$. The outer edge of the accretion flow is at a radius $r=r_{\text {out }}$, where $r$, the radius in 
Schwarzschild units, is related to the physical radius $R$ by

$$
r=\frac{R}{R_{S}}, \quad R_{S}=\frac{2 G M}{c^{2}} .
$$

The angular momentum vector of the binary is inclined at an angle $i$ to the line-of-sight. For many astrophysical sources we have direct estimates of $M, r_{\text {out }}$ and $i$ from the observations. However, $\dot{M}$ is generally not known and needs to be adjusted so as to fit the observed spectrum.

Following NMY, we model the accretion flow by means of two zones separated at a transition radius $r_{\mathrm{tr}}$. For $r<r_{\mathrm{tr}}$, we assume that the gas is in the form of an optically thin two-temperature ADAF. For $r>r_{\text {tr }}$, the accretion occurs primarily in the form of a thin accretion disk, but with part of the mass accreting via a corona above the disk. In our picture, the accretion stream from the companion star arrives at the disk in a more or less cool state and initially forms a standard thin disk. As the accretion proceeds inward, gas evaporates continuously from the surface of the disk into the corona, so that with decreasing radius more and more of the gas shifts from the disk to the corona. By $r=r_{\text {tr }}$, the entire accretion flow is transformed completely into the corona, and the coronal flow continues inward as a pure ADAF. As far as the physics of the flow is concerned, we do not make any distinction between the ADAF and the corona. We view the corona merely as another component of the ADAF, except that it happens to be at the same radius as an equatorial thin disk.

The evaporation from thin disk to corona probably occurs by a process similar to that described by Meyer \& Meyer-Hofmeister (1994) or Honma (1995). However, there are uncertainties still in the precise mechanism and it is not possible to estimate from first principles either the rate of evaporation or the precise location of the transition radius. We therefore model the evaporation in an empirical way and assume that the mass accretion rate in the corona $\left(\dot{M}_{c}\right)$ and the disk $\left(\dot{M}_{d}\right)$ vary with radius $r$ as

$$
\dot{M}_{c}=\dot{M}\left(\frac{r_{\mathrm{tr}}}{r}\right), \quad \dot{M}_{d}=\dot{M}-\dot{M}_{c}, \quad r>r_{\mathrm{tr}} .
$$

The functional form we have assumed for $\dot{M}_{c}$ is fairly arbitrary, but it is probably not unreasonable. In any case, the results presented in this paper are completely insensitive to the specific choice made in equation (2.1.2), as we show in $\S 3.3$.

We estimate the transition radius $r_{\text {tr }}$ on the basis of the largest velocity $v_{\max }$ seen in the $\mathrm{H} \alpha$ emission line from the thin accretion disk (cf. NMY):

$$
r_{\mathrm{tr}}=\frac{1}{2}\left(\frac{c \sin i}{v_{\max }}\right)^{2} \text {. }
$$


This completes the description of the binary system and the geometry of the flow. In addition, we need three parameters to describe the properties of the accreting gas in the $\mathrm{ADAF}$ and the corona:

1. We model viscosity by means of the standard $\alpha$ prescription (cf. Frank, King \& Raine 1992) and write the kinematic viscosity coefficient as

$$
\nu=\alpha \frac{c_{s}^{2}}{\Omega_{K}}
$$

where $c_{s}$ is the isothermal sound speed $(p / \rho)$ and $\Omega_{K}$ is the Keplerian angular velocity. We assume that the parameter $\alpha$ is independent of $r$. In most of our models, we set $\alpha=0.3$.

2. We assume that the accreting gas in the ADAF and corona consists of a mixture of particles and magnetic fields in rough equipartition. Thus, we write the pressure due to the two components as

$$
p_{\text {gas }}=\beta p_{\text {tot }}, \quad p_{\text {mag }}=(1-\beta) p_{\text {tot }},
$$

with $\beta$ taken to be independent of $r$. We do not consider radiation pressure, which is easily shown to be negligible in these flows (Narayan \& Yi 1995b). In our calculations, we generally set $\beta=0.5$, but we also show one model with $\beta=0.95$. In principle, if the macroscopic viscosity is entirely due to magnetic fields, we may expect $\alpha$ and $\beta$ to be related to each other. For instance, according to Hawley (1996),

$$
\alpha \sim \frac{1}{2} \frac{p_{\mathrm{mag}}}{p_{\mathrm{tot}}}=\frac{1-\beta}{2} .
$$

Our standard parameter set, $\alpha=0.3, \beta=0.5$, is compatible with this scaling.

3. We include a third parameter $\delta$, which describes the fraction of the viscous energy which goes directly into electrons. Various heuristic arguments (e.g. Shapiro et al. 1976, Phinney 1981, Rees et al. 1982) suggest that viscous dissipation primarily heats up ions and that only a fraction $\sim\left(m_{e} / m_{i}\right)$ of the energy goes directly into electrons. With this in mind, we normally set $\delta=10^{-3}$. However, we also try larger values of $\delta$ in order to test the sensitivity of the results to this parameter.

\subsection{Dynamics and Energy Balance of the ADAF and Corona}

We employ a logarithmically spaced radial grid to describe the properties of the ADAF, the corona, and the thin disk. The cell edges are at radii $\left\{l_{j}\right\}$, where $l_{1}=1$ (black 
hole event horizon) and $l_{n}=r_{\text {out }}$ (outer edge). The mid-points of the cells are denoted by $\left\{r_{j}\right\}$, with $\log r_{j}=\left(\log l_{j-1}+\log l_{j}\right) / 2$. Cells are identified by the index $j$ of the mid-point, which in this scheme ranges from 2 to $n$. Typically, we have ten cells per decade of radius. We do not make a distinction between the ADAF and the corona, but treat them as a single entity. The only exception to this statement is that we allow $\dot{M}$ to change with $r$ in the corona (cf. eq. 2.1.2), whereas it is independent of $r$ in the pure ADAF zone.

We model the ADAF as a set of nested spherical shells. In order to allow for the flattening of the density profile, we truncate each shell near the pole in the manner discussed in Appendix A. Thus, the shell at $r_{j}$ extends from polar angle $\theta_{H, j}$ to $\pi-\theta_{H, j}$, where $\theta_{H, j}$ is calculated from the local sound speed and angular velocity of the gas (Appendix A). For simplicity, we assume that the gas density is constant within each radial shell.

We take the radial velocity $v(r)$, angular velocity $\Omega(r)$ and isothermal sound speed $c_{s}(r)$ from the numerical global solutions calculated by Narayan et al. (1997) (see also Chen et al. 1997). Each model satisfies the basic conservation laws of mass, radial momentum, angular momentum and energy, along with consistent boundary conditions at the inner and outer edges of the flow. On the outside, the solutions match on to a self-similar form (Narayan \& Yi 1994), while on the inside the gas makes a sonic transition and falls supersonically into the black hole, with a zero-torque condition at the horizon (see Narayan et al. 1997 for details). The models assume a pseudo-Newtonian form of the gravitational potential which mimics some of the properties of a Schwarzschild black hole (Paczyński \& Wiita 1980).

Each global model is uniquely specified by the value of the viscosity parameter $\alpha$, the ratio of specific heats $\gamma$ of the accreting gas, and a function $f(r)$ which describes the nature of the energy advection. For a plasma with a constant $\beta$ and an isotropically tangled magnetic field, Esin (1996) has shown that the effective $\gamma$ is given by

$$
\gamma=\frac{8-3 \beta}{6-3 \beta} .
$$

(Note that this is different from the value quoted in Narayan \& Yi 1995b.) The function $f(r)$ describes at each radius $r$ the fraction of the viscously dissipated energy which is advected radially with the gas. For the quiescent SXTs which we consider in this paper, the radiative efficiency is extremely low and so $f(r)$ is practically equal to unity at all radii. We set $f(r)=1$ in calculating the global models, an excellent approximation for the calculations presented here.

The global solution specifies the values of $v_{j}, \Omega_{j},(d \Omega / d r)_{j}$, and $c_{s, j}$ at the mid-points 
of the cells of our model. Mass conservation directly gives the density:

$$
\rho_{j}=\frac{\dot{M}(r)}{4 \pi r_{j}^{2} v_{j} \cos \theta_{H, j}} .
$$

Note that $\dot{M}(r)$ is equal to the full $\dot{M}$ for $r_{j}<r_{\text {tr }}$, but is equal to the coronal $\dot{M}_{c}\left(r_{j}\right)$ for $r_{j} \geq r_{\mathrm{tr}}$ (cf. eq. 2.1.2). The viscous heating rate per unit volume is given by

$$
q_{j}^{+}=\rho_{j} \nu_{j}\left(r \frac{d \Omega}{d r}\right)_{j}^{2}, \quad \nu_{j}=\alpha \frac{c_{s, j}^{2}}{\Omega_{K, j}},
$$

where $\nu_{j}$ is the kinematic viscosity coefficient in the $j$ th cell (cf. eq. 2.1.4). The heating over the entire shell is then

$$
Q_{j}^{+}=q_{j}^{+} V_{j}, \quad V_{j}=\frac{4 \pi}{3}\left(l_{j}^{3}-l_{j-1}^{3}\right) \cos \theta_{H, j}
$$

Since we model the accreting gas as a two-temperature plasma (Shapiro et al. 1976), we need to determine at each radius the ion temperature $T_{i}$ and electron temperature $T_{e}$. By the definition of $\beta$, we immediately have one constraint on the temperatures:

$$
\beta \rho c_{s}^{2}=p_{\text {gas }}=\frac{\rho k T_{i}}{\mu_{i} m_{u}}+\frac{\rho k T_{e}}{\mu_{e} m_{u}},
$$

where $\mu_{i}=1.23$ and $\mu_{e}=1.14$ (Narayan \& Yi 1995b). A second relation is obtained via the energy balance of the electrons:

$$
\delta Q_{j}^{+}+Q_{j}^{\mathrm{ie}}=Q_{j}^{-}
$$

All quantities in this equation refer to shell $j$ and are integrated over the shell. The first term on the left is the direct viscous heating of electrons (recall the definition of $\delta$ in $\S 2.1)$. The second term is the heating of electrons via Coulomb collisions with the ions. Stepney \& Guilbert (1983) have given a convenient expression for this heating rate as a function of the densities and temperatures of the electrons and ions. The term on the right is the radiative cooling of the gas in the shell, which is computed by the methods described in $§ 2.3$. By simultaneously solving equations (2.2.5) and (2.2.6) in each radial shell we determine $T_{i, j} \equiv T_{i}\left(r_{j}\right)$ and $T_{e, j} \equiv T_{e}\left(r_{j}\right)$ in the various shells. The solution is obtained iteratively.

\subsection{Radiative Transfer, Cooling, and Spectrum}

The bulk of the effort goes into calculating the radiative properties of the ADAF and corona. Because of the non-local nature of the radiative interactions (especially Compton 
scattering), we use the iterative scattering method (Poutanen \& Svensson 1996, Sunyaev $\&$ Titarchuk 1985), which is related to the $\Lambda$ iteration method (Mihalas 1978). This method allows us to build up the local radiation field at each point in the flow iteratively and at the same time to calculate the rate of cooling $\left(Q_{j}^{-}\right)$of the accreting gas. As a by-product the calculation also gives the spectrum seen by an observer at infinity.

A few approximations are made in the version of the calculations described below. This is a non-relativistic code, as dictated by the fact that we use a pseudo-Newtonian potential (Paczyński \& Wiita 1980) to calculate the global flow solution. As part of this approximation, we neglect gravitational redshift, Doppler boosts, and ray deflections. For advection-dominated flows the first two effects tend largely to cancel each other, and one should either include both self-consistently or not include either. We have chosen the latter alternative. Abramowicz et al. (1996) have recently computed global solutions in Kerr geometry and it should soon be possible to develop relativistically consistent radiative models. Another simplification is that we set the radial velocity and density to be independent of $\theta$ (the constancy of $v$ is implicit in eq. 2.2.2).

\subsubsection{Scattering Probability Matrices}

Compton scattering is an important, often dominant, process in ADAFs. Since the accreting gas is optically thin, scattering is extremely non-local, and photons emitted at one radius are capable of being scattered at practically any other radius. To deal with this we calculate the following three matrices which describe the properties of the scattering:

$P_{j k}^{a a}$ : The probability that a photon emitted by the ADAF (or corona, recall we make no distinction) in shell $j$ is scattered by an ADAF electron in shell $k$.

$P_{j k}^{\mathrm{ad}}$ : The probability that a photon emitted by the ADAF in shell $j$ irradiates ring $k$ of the thin disk. We assume that the irradiating flux is completely absorbed by the disk.

$P_{j k}^{\text {da }}$ : The probability that a photon emitted by ring $j$ of the thin disk is scattered in shell $k$ of the ADAF.

In principle, each of these matrices is a function of the frequency $\nu$ of the photon since the scattering cross-section declines with increasing photon energy in the KleinNishina regime. We ignore the frequency dependence in calculating the $P_{j k}$, using the non-relativistic Thomson cross-section $\sigma_{T}$ for the cross-section per electron. The correct cross-section is, however, automatically included when doing the actual Comptonization calculation (see $§ 2.3 .4$ below). The error due to this approximation is therefore minor. 
We assume that the radiation emitted at each point in the flow is locally isotropic. This assumption is valid for the synchrotron and bremsstrahlung radiation (except for the effect of Doppler beaming which is ignored in this treatment), but is a simplification for Compton scattering. The reason is that the incident radiation field at each point of the flow is in general anisotropic, and so the scattered radiation too would have some residual anisotropy (though less than in the incident field). The anisotropy is easily included in the calculations by generalizing the $P_{j k}$ to $P_{j k, l m}$ where $l$ and $m$ refer to the orientations of the pre- and post-scattered photon. We have avoided this generalization in the interests of computational speed.

The matrix elements $P_{j k}^{\mathrm{aa}}$ and $P_{j k}^{\mathrm{ad}}$ are computed by shooting a large number of rays out of each shell $j$ and following their scattering histories. The points of origin of the rays are distributed uniformly in $\cos \theta$ within each shell; typically we choose $n_{\theta}=6$ different values of $\cos \theta$. At each point of origin, we select a set of ray directions $\left\{\chi_{l}\right\}$ where $\chi$ is the angle between the ray direction and the negative of the local radius vector. Because the ADAF extends over many decades of radius, the set of $\left\{\chi_{l}\right\}$ has to be selected with care so that interactions between all pairs of shells are included. The specific choice we make is the following set of backward oriented rays:

$$
\sin \chi_{l}=r_{l+1} / r_{n}, \quad l=1,2, \cdots, n-2 .
$$

This set is augmented with a few additional rays in sideways and forward directions to fill up the entire $4 \pi$ solid angle. Typically, we end up with a set of about 50 values of $\chi_{l}$. The weights of the rays are chosen so as to reflect the solid angle covered by each. Finally, for each point of origin and each $\chi_{l}$, we choose $n_{\phi}$ different rays corresponding to different azimuthal angles relative to the local radius vector; usually $n_{\phi}=6$. Thus, in total, we have over $10^{3}$ rays per radial shell.

We calculate the trajectory of each ray and compute the distances traversed by the ray in various shells (including the shell of origin). Since we know the electron densities in the shells (from $\rho_{j}$, eq. 2.2.2), we can compute the differential Thomson cross-sections and can thereby calculate the fraction of the energy in the ray which is lost by scattering in each shell. These quantities are accumulated in $P_{j k}^{\text {aa }}$ with the weight appropriate to the ray. If a ray intersects the thin disk at any point, it is assumed that all the residual energy in the ray is absorbed by the particular local ring segment $k$ of the disk. In this case, the matrix element $P_{j k}^{\text {ad }}$ is updated. Note that we do not include electron-positron pairs in the model. At the low mass accretion rates considered in this paper pairs are quite negligible (cf. Bjornsson et al. 1996, Kusunose \& Mineshige 1996).

In an exactly analogous fashion, we shoot rays off the surfaces of the various rings $j$ in the thin disk and follow their histories to calculate the matrix elements $P_{j k}^{\mathrm{da}}$. 
As an independent calculation, we shoot rays from the $\mathrm{ADAF} /$ corona and the thin disk at the specific inclination angle $i$ of the system and calculate the following quantities: $E_{j}^{\mathrm{a}}$ : The probability that a photon emitted in shell $j$ of the ADAF or corona at an angle $i$ to the spin axis escapes unscattered to infinity.

$E_{j}^{\mathrm{d}}$ : The probability that a photon emitted in ring $j$ of the thin disk at an angle $i$ to the spin axis escapes unscattered to infinity.

\subsubsection{Bremsstrahlung Emission}

The net bremsstrahlung cooling per unit volume, $q_{\text {brem }}^{-}$, of a thermal plasma has been calculated by Stepney \& Guilbert (1983) and Svensson (1982) (with some slight modifications introduced by Narayan \& Yi 1995b). The luminosity from shell $j$ is thus given by

$$
L_{\mathrm{brem}, j}(\nu) d \nu=q_{\mathrm{brem}, j}^{-} V_{j}\left(\frac{h}{k T_{e, j}}\right) \exp \left(-\frac{h \nu}{k T_{e, j}}\right) \bar{g}_{\mathrm{ff}}(\nu) d \nu,
$$

where $\bar{g}_{\mathrm{ff}}(\nu)$ is a velocity averaged Gaunt factor, which we take from Novikov \& Thorne (1973). We normalize $\bar{g}_{\mathrm{ff}}$ so that the integral of $L_{\mathrm{brem}, j} d \nu$ is equal to $q_{\mathrm{brem}, j} V_{j}$, the net cooling of the shell due to bremsstrahlung emission.

Free-free absorption is extremely small in all our ADAF models and is neglected.

Note: we consider below a number of spectral densities similar to $L_{\mathrm{brem}, j}(\nu)$. All of these are functions of $\nu$ and have units of ergs $\mathrm{s}^{-1} \mathrm{~Hz}^{-1}$. To simplify the notation we will omit the argument $(\nu)$ unless it is essential.

\subsubsection{Synchrotron Emission}

The electrons in ADAF models are typically quasi-relativistic $\left(k T_{e} \sim m_{e} c^{2}\right)$ and therefore emit cyclo-synchrotron radiation in the local magnetic field. The cyclosynchrotron emissivity $j_{\text {synch }}(\nu)$ of a thermal plasma has been worked out by a number of authors in various limits (Pacholczyk 1970, Petrosian 1981, Takahara \& Tsuruta 1982, Mahadevan, Narayan \& Yi 1996), and convenient fitting formulae for the general case are presented by Mahadevan et al. (1996). The emission from shell $j$ in the optically thin limit is thus easily calculated: $j_{\text {synch }, j}(\nu) V_{j}$. The main complication is that the synchrotron emission is self-absorbed. We allow for this as follows.

We step outwards, starting with the innermost shell, and calculate for each shell $j$ 
the net outgoing synchrotron radiation $L_{\text {synch }, j}$ due to all shells interior to and including shell $j$. To understand how we calculate $L_{\text {synch }, j}$, consider the changes that occur when we step from shell $j$ to shell $j+1$ :

(i) A fraction $P_{j, j+1}^{\mathrm{aa}}$ of the outgoing $L_{\mathrm{synch}, j}$ is scattered in shell $j+1$.

(ii) The shell $j+1$ emits synchrotron radiation with a net luminosity of $j_{\text {synch }, j+1} V_{j+1}$. This is added to the previous luminosity.

(iii) Some part of the radiation is absorbed by the gas. We handle the absorption in an approximate fashion by truncating $L_{\text {synch }, j+1}$ whenever it exceeds the local blackbody luminosity. In the Rayleigh-Jeans limit, the maximum luminosity that can exit shell $j+1$ at frequency $\nu$ is

$$
L_{\max , j+1}(\nu) d \nu=2 k T_{e, j+1} \frac{\nu^{2}}{c^{2}} A_{j+1}^{\mathrm{d}} d \nu, \quad A_{j+1}^{\mathrm{a}}=2 \pi l_{j+1}^{2}\left(2 \cos \theta_{H, j+1}+\sin ^{2} \theta_{H, j+1}\right),
$$

where $A_{j+1}^{\mathrm{a}}$ is the surface area of shell $j+1$ (including the polar caps).

Thus, we have the following recursion relation for calculating the synchrotron luminosity exiting shell $j+1$ :

$$
L_{\mathrm{synch}, j+1}=\operatorname{Min}\left[\left(1-P_{j, j+1}^{\mathrm{aa}}\right) L_{\mathrm{synch}, j}+j_{\mathrm{synch}, j+1} V_{j+1}, L_{\mathrm{max}, j+1}\right] \equiv L_{\mathrm{synch}, j}+L_{s, j+1} .
$$

Starting with $L_{\mathrm{synch}, 1}=0$, this recursion allows us to estimate the synchrotron luminosity $L_{\text {synch }, j}$ exiting each succeeding shell $j$ outwards from the center.

The quantity $L_{s, j+1}$ on the right hand side of equation (2.3.4) represents the net luminosity of shell $j+1$ alone (as distinct from $L_{\text {synch }, j+1}$ which is the luminosity of all shells from 2 to $j+1$ ). The integral of $L_{s, j+1} d \nu$ gives the cooling of shell $j+1$ as a result of synchrotron radiation.

\subsubsection{Compton Scattering}

Consider a photon of frequecy $\nu^{\prime}$ that is Compton-scattered once by thermal electrons at a temperature $T_{e}$. Define $C\left(\nu^{\prime}, \nu ; T_{e}\right) d \nu$ to be the isotropically averaged probability distribution of the frequency $\nu$ of the scattered photon. This distribution has been calculated by Jones (1964), with corrections published by Coppi \& Blandford (1990). The formulae given in these papers are exact and include relativistic corrections corresponding to the Klein-Nishina regime.

In each shell $j$, we define $L_{\mathrm{C}, \mathrm{in}, j}$ to be the net radiative energy which is Comptonscattered per second, and $L_{\mathrm{C}, \text { out }, j}$ to be the net output luminosity in the scattered photons. 
By the definition of $C\left(\nu^{\prime}, \nu ; T_{e}\right)$, we have

$$
L_{\mathrm{C}, \text { out }, j} d \nu=\left[\int L_{\mathrm{C}, \mathrm{in}, j}\left(\nu^{\prime}\right) C\left(\nu^{\prime}, \nu ; T_{e, j}\right) d \nu^{\prime}\right] d \nu .
$$

This relation allows us to calculate the contribution of each shell to Comptonization, provided we know $L_{\mathrm{C}, \mathrm{in}, j}$. The total outgoing luminosity from shell $j$, including the contributions from synchrotron and bremsstrahlung emission, is thus

$$
L_{\mathrm{out}, j}=L_{\mathrm{brem}, j}+L_{s, j}+L_{\mathrm{C}, \mathrm{out}, j} .
$$

To calculate $L_{\mathrm{C}, \mathrm{in}, j}$, we note that any radiation emitted in any shell $k$ of the ADAF or ring $l$ of the thin disk has a certain probability of being scattered in shell $j$, the probability being given by the various matrices discussed in $§ 2.3 .1$. Therefore,

$$
L_{\mathrm{C}, \mathrm{in}, j}=\Sigma_{k} P_{k j}^{\mathrm{aa}} L_{\mathrm{out}, k}+\Sigma_{l} P_{l j}^{\mathrm{da}} L_{\mathrm{disk}, l},
$$

where $L_{\text {disk }, l}$ is the spectral luminosity of ring $l$ of the thin disk. Equation (2.3.7) and eqs. (2.3.8) and (2.3.10) below close the loop for calculating the effect of Comptonization. The equations are, however, strongly coupled among different shells and rings, and require an iterative technique of solution.

\subsubsection{Outer Thin Accretion Disk}

The outer disk has two sources of energy: (i) its own internal viscous dissipation, and (ii) irradiation from the ADAF and corona. By the standard equations of thin accretion disk theory (cf. Frank et al. 1992), we have the following expression for the effective temperature of ring $j$ of the disk:

$$
\sigma T_{\mathrm{eff}, j}^{4} A_{j}^{\mathrm{d}}=\frac{3 G M \dot{M}}{2 R_{S}}\left\{\frac{1}{l_{j-1}}\left[1-\frac{2}{3}\left(\frac{r_{\mathrm{tr}}}{l_{j-1}}\right)^{1 / 2}\right]-\frac{1}{l_{j}}\left[1-\frac{2}{3}\left(\frac{r_{\mathrm{tr}}}{l_{j}}\right)^{1 / 2}\right]\right\}+\Sigma_{k} P_{k j}^{\mathrm{ad}} L_{\mathrm{out}, k},
$$

where

$$
A_{j}^{\mathrm{d}}=4 \pi\left(l_{j}^{2}-l_{j-1}^{2}\right)
$$

is the surface area of the ring, counting both the top and bottom surfaces. The second term on the right of equation (2.3.8) is the net luminosity absorbed by the ring via irradiation from the ADAF and corona.

We assume that the disk emits as a blackbody. Thus, the outgoing luminosity from ring $j$ is written as

$$
L_{\text {disk }, j} d \nu=\frac{A_{j}^{\mathrm{d}}\left(2 h \nu^{3} / c^{2}\right) d \nu}{\exp \left(h \nu / k T_{\mathrm{eff}, j}\right)-1} .
$$


Note that in our model we do not need to assume a specific value of the viscosity parameter $\alpha_{d}$ in the outer disk. All we require is that the emission from the disk has a locally blackbody form, and this is a valid approximation so long as $\alpha_{d}$ in the disk is small enough to make the gas optically thick in the vertical direction.

In our calculations, we assume that all the irradiating flux is absorbed by the disk. Actually, a fraction of the flux is reflected and only the remainder is absorbed. The error we make by ignoring reflection is negligible since the contribution of the disk to the calculated spectra is very small $(\S 3.2)$.

\subsubsection{Iterative Scattering}

The sychrotron and bremsstrahlung luminosities, $L_{s, j}$ and $L_{\mathrm{brem}, j}$, can be directly calculated once the electron temperatures $T_{e, j}$ are given. However, the Comptonized radiation is less straightforward since the output from each shell acts as an input to other shells. A convenient technique to handle this situation is the iterative scattering method or $\Lambda$ iteration method (Poutanen \& Svensson 1996, Mihalas 1978), where the radiative transfer equation is solved for each scattering order. (The technique is very efficient at low optical depths, as in the present work, but is less useful when the optical depth is greater than a few.) Operationally, for our problem, the iterative scattering method consists of iterating on equation (2.3.5) until convergence. At the first iteration, we set $L_{\mathrm{C}, \text { out }, j}=0$ and calculate the various $L_{\mathrm{C}, \mathrm{in}, j}$ via equations (2.3.6)-(2.3.10). Using these $L_{\mathrm{C}, \mathrm{in}, j}$, we calculate new estimates of $L_{\mathrm{C}, \mathrm{out}, j}$. Then, we use these to obtain new estimates of $L_{\mathrm{C}, \mathrm{in}, j}$, calculate new $L_{\mathrm{C}, \mathrm{out}, j}$, etc. until convergence.

The net radiative cooling in each shell is given by

$$
Q_{j}^{-}=\int\left(L_{\mathrm{out}, j}-L_{\mathrm{C}, \mathrm{in}, j}\right) d \nu
$$

This quantity is used in equation (2.2.6) to solve for the ion and electron temperatures in the various shells.

Once the temperatures and spectra have converged, we obtain the spectral flux $F(\nu) d \nu$ seen by an observer at distance $D$ as follows,

$$
4 \pi D^{2} F(\nu)=\Sigma_{j} E_{j}^{\mathrm{a}} L_{\mathrm{out}, j}+\Sigma_{k} E_{k}^{\mathrm{d}} L_{\mathrm{disk}, k}
$$

The escape probabilities $E_{j}^{\mathrm{a}}$ and $E_{k}^{\mathrm{d}}$ correspond to the particular inclination $i$ of the system, as described in $§ 2.3 .1$. 


\section{V404 Cyg in Quiescence}

We have applied the above model to the SXT source V404 Cyg in quiescence. §3.1 describes our analysis of ASCA observations of the source in the X-ray band and $\S 3.2$ describes observations in other wavelength bands. $\S 3.3$ compares the observations with theoretical model spectra and shows that the ADAF model provides an excellent fit to the data. Finally, $§ 3.4$ describes a re-analysis of the source A0620-00.

\subsection{Analysis of archival X-ray data}

V404 Cyg was observed by ASCA on 9-10 May 1994 for a total exposure time of about $40 \mathrm{ksec}$ with the GIS detectors and $35.5 \mathrm{ksec}$ with the SIS detectors. The results of these observations are not currently available in the literature. We therefore retrieved the data from the HEASARC archives and performed a spectral analysis in order to test the predictions of the ADAF model. The good data have been selected following the procedure described in Day et al. (1995)

The spectral analysis was somewhat difficult because the source flux is comparable to the background flux. Therefore, special care was taken with the background subtraction.

For the two GIS detectors, we determined the background in two ways: using observations of blank fields at high latitudes (Method I), and using data extracted from an annular region around the source (Method II). Given the low Galactic latitude of the source ( $\mathrm{b}=$ $-2.2^{\circ}$ ), unresolved emission from the Galactic plane may contribute to the source flux; therefore we prefer Method II for the GIS observations. (The results of our analysis, however, show that Method I gives results that are fully consistent with Method II). For the GIS detectors we did the full analysis using different extraction radii for the source region (4, 5 and 6 arcmin). We found that the optimum radius (the one which maximizes the signal to noise ratio) was 5 arcmin.

For the two SIS detectors, we determined the background using data from high-latitude blank fields. We used the November 1994 release of the background files provided by the ASCA Guest Observer facility at HEASARC. For these detectors we fixed the extraction radius for the source region at 3.5 arcmin. V404 Cyg is clearly detected in the SIS detectors from about 0.7 to $8.5 \mathrm{keV}$ with an average count rate of $0.0102 \pm 0.0008 \mathrm{cts} \mathrm{s}^{-1}$. The average count rate in the GIS detectors is very similar: $0.0107 \pm 0.0015$ cts s $^{-1}$ over the same energy band. In order to use the $\chi^{2}$ statistic in the model fitting, we grouped the raw SIS spectra so that each spectral bin contained at least 20 photons (40 photons for the GIS spectra). We found that the spectra determined using the SIS0, SIS1, GIS2 
and GIS3 detectors were individually consistent with each other within the errors. Thus, we combined the four individual spectra to obtain the final spectrum.

We fitted the data to a simple power law (PL) model with two free parameters: a single photon index, $\alpha$, and a column density $\mathrm{N}_{\mathrm{H}}$. This model with $\alpha=2.1$ and $\mathrm{N}_{\mathrm{H}}=$ $1.1 \times 10^{22} \mathrm{~cm}^{-2}$ provides an excellent fit to the ASCA data $\left(\chi_{\nu}^{2}=1.0\right.$ for 105 d.o.f; Table 1). A comparably good fit is provided by a thermal bremsstrahlung (TB) model with a temperature of $4.7 \mathrm{keV}$ because the shapes of the TB and PL models are similar over the energy range $0.7-8.5 \mathrm{keV}$. A blackbody (BB) model provides a poorer fit than either the PL or the TB model; nevertheless, a BB model cannot be ruled out because the difference in $\chi_{\nu}^{2}$ is not statistically significant. (The probability that $\chi_{\nu}^{2}$ is larger than the value derived from the PL or TB fit is $49 \%$, whereas it is $\sim 15 \%$ in the case of the BB fit.)

An inspection of the residuals for the BB fit indicates, however, that above $5 \mathrm{keV}$ the data points lie systematically above the model; moreover, interstellar absorption is clearly a dominant factor below about $2 \mathrm{keV}$, which is near the peak of the BB model. Consequently, we re-fitted the data above $2 \mathrm{keV}$ excluding the parameter $\mathrm{N}_{\mathrm{H}}$ and found that the BB model gives a poor description of the data: $\chi_{\nu}^{2}=1.4$ (62 d.o.f.) with a corresponding probability of only $2 \%$. On the other hand, the PL fit is still acceptable $\left(\chi_{\nu}^{2}=1.1\right)$, and gives a photon index of $1.68_{-0.29}^{+0.27}$, consistent with the value derived from the fit between 0.7 and $8.5 \mathrm{keV}$. Therefore, although the BB model cannot be completely ruled out by the current data, these results suggest that it is not the correct description of the source spectrum.

The best fit results for the three models are listed in Table 1, and the combined GIS+SIS unfolded spectrum for the PL model is shown in Figure 1. In Figure 2a we show the allowed grid of variations of column density $\mathrm{N}_{\mathrm{H}}$ and photon index $\alpha$. Assuming the PL model, the 1-10 keV unabsorbed X-ray flux is $8.2 \times 10^{-13} \mathrm{ergs}^{-2} \mathrm{sm}^{-1}$, corresponding to a luminosity of $1.2 \times 10^{33} \mathrm{ergs} \mathrm{s}^{-1}$.

The error box shown in Figures $3-5$ is given at the $2 \sigma$ level and was derived as follows. The upper/lower curves of the box are defined as the maximum/minimum fluxes allowed by any combinations of $\alpha$ and its corresponding normalization on the $2 \sigma$ contour in Figure 2b. The box extends from 0.5 to $10 \mathrm{keV}$. The "throat" of the box represents the most accurately measured region of the spectrum. The center of the throat corresponds to: $E=3.5 \mathrm{keV}$ (i.e. $\log \nu=17.93$ ), and $\nu F_{\nu}=3.56 \times 10^{-13} \mathrm{ergs} \mathrm{s}^{-1} \mathrm{~cm}^{-2}$ (i.e. $\left.\log \left(\nu F_{\nu}\right)=-12.45\right)$. The models described in $\S 3.2$ were fitted to this flux measurement.

V404 Cyg was observed with the ROSAT satellite and the PSPC detector by Wagner et al. (1994). They fitted the data with a power-law model and found a photon index 
of $\alpha \sim 7$ (no uncertainty given). NMY re-analyzed these ROSAT data and found the following value for the power-law index, $\alpha=4.0_{-1.5}^{+1.9}$, which is consistent with the ASCA value given in Table 1 . The ASCA results reported here are superior to the ROSAT results and supersede them primarily because ASCA has a much greater useful bandwidth for observing V404 Cyg: 0.7-8.5 keV vs. $0.7-2.4 \mathrm{keV}$.

\subsection{Observations of V404 Cyg in Other Wavelength Bands}

The dereddened B, V and R magnitudes of V404 Cyg, and the fraction of the total flux in these bands contributed by the accretion disk are given by Casares et al. (1993). From these data NMY extracted the following flux estimates for the accretion disk in the $\mathrm{B}, \mathrm{V}$ and $\mathrm{R}$ bands: $\log \left(\nu F_{\nu}\right)=-11.256,-11.293,-11.368$, respectively at $\log (\nu)=14.834,14.737,14.632\left(\nu\right.$ in $\mathrm{Hz}, F_{\nu}$ in $\left.\operatorname{ergs~s}^{-1} \mathrm{~cm}^{-2} \mathrm{~Hz}^{-1}\right)$.

V404 Cyg is significantly reddened $\left(A_{V} \approx 4.0 \mathrm{mag}\right)$. Therefore, it is not easy to measure the UV flux. However, following the approach used by Marsh, Robinson \& Wood (1994) for A0620-00, we can obtain a limit on the EUV flux. The HeII 4686 emission line is absent in the sensitive blue spectrum of V404 Cyg shown in Figure 1 (top) of Casares et al. (1993). A reddened version of this spectrum in digital form was kindly provided to us by J. Casares. We dereddened the spectrum using $A_{\mathrm{V}}=4.0$ mag (Cardelli, Clayton \& Mathis 1989), which admirably flattened a $300 \AA$ interval of the continuum centered on HeII 4686. We assumed that the full width at zero intensity (FWZI) of the $\lambda 4686$ line is $40 \AA$ (which is approximately the FWZI of the $\mathrm{H}_{\beta}$ emission line), and measured the total intensities in three adjacent $40 \AA$ bands with the central band centered on $\lambda 4686$. These three intensities differ by somewhat less than the uncertainties in the individual bands, which we estimated from the rms fluctuations in the (1.4 $\AA$-wide) pixels to be $1.2 \%$. Using this value as an estimate of the standard deviation in the intensity of a $40 \AA$ band, we obtain the following upper limit on the equivalent width of the line: $\operatorname{EW}(\lambda 4686)$ $<1.4 \AA(3 \sigma)$.

We use the absence of HeII 4686 to constrain the EUV flux from either an inner accretion disk or from an ADAF region. (For details see the derivation for A0620-00 by Marsh et al. 1994). We assume that every photon in the energy range $55<\mathrm{h} \nu<280 \mathrm{eV}$ photoionizes HeI just once, and that a fraction $\epsilon=0.2$ of the subsequent recombinations produce a HeII 4686 photon. The fraction $\alpha$ of the EUV photons that photoinize HeI is the same as the fraction of the sky (as viewed from the compact object) that is covered by the accretion disk and by the secondary star, which we estimate to be 0.008 (Shahbaz et

al. 1994) and $\approx 0.02$ (Frank, King, \& Raine 1992), respectively. Thus, we take $\alpha \approx 0.03$. 
Here we have made the reasonable assumption that both the annular disk in our ADAF model and the complete thin disk stop about the same fraction of the EUV photons. The dereddened continuum flux at B is $f_{\nu} \approx 3.4 \times 10^{-26} \mathrm{ergs} \mathrm{s}^{-1} \mathrm{~cm}^{-2} \mathrm{~Hz}^{-1}$ (Casares et al. 1993). Assuming an average EUV photon energy of $\overline{\mathrm{E}}=100 \mathrm{eV}$ and $\mathrm{D}=3.5 \mathrm{kpc}$, we can now translate the limit on the HeII 4686 photon flux into a $3 \sigma$ limit on the EUV luminosity:

$$
L_{\mathrm{EUV}}<2 \overline{\mathrm{E}} \frac{\mathrm{EW}}{\lambda} \frac{f_{\nu}}{h} \frac{4 \pi D^{2}}{\alpha \epsilon}=1.2 \times 10^{35} \mathrm{ergs} \mathrm{s}^{-1} .
$$

The corresponding limit on the average flux density in the $55-280 \mathrm{eV}$ band is $\log \left(\nu F_{\nu}\right)<-10.44(3 \sigma)$.

\subsection{Models of V404 Cyg in Quiescence}

V404 Cyg is a well-studied source and many of the system parameters are reasonably well constrained. We choose a black hole mass $M=12 M_{\odot}$ and inclination $i=56^{\circ}$ (Shahbaz et al. 1994). Given the size of the Roche lobe around the black hole, we estimate the outer radius of the accretion flow to be $r_{\text {out }}=10^{5}$. The $\mathrm{H} \alpha$ line of V404 Cyg shows a maximum velocity of $1140 \mathrm{~km} \mathrm{~s}^{-1}$ (NMY; Casares et al. 1993), which corresponds to a transition radius $\log \left(r_{\text {tr }}\right)=4.4$ (cf. eq. 2.1.3). The mass accretion rate $\dot{M}$ is not known. In each of the models described below, we have adjusted $\dot{M}$ so as to fit the X-ray flux in the "throat" of the ASCA error box, as described in §3.1. The fluxes corresponding to the model spectra are calculated assuming a distance $D=3.5 \mathrm{kpc}$ in equation (2.3.12) (Wagner et al. 1992, Shahbaz et al. 1994).

This still leaves $\alpha, \beta$ and $\delta$ undetermined. In much of our previous work we have chosen values of $\alpha$ in the range 0.1 to 1 . There are two reasons for this. First, theoretical models of the dwarf nova instability strongly suggest that accretion disks in cataclysmic variables have low values of $\alpha \sim 0.01$ when they are in a cool state and larger values $\sim 0.1$ when they switch to a hot state (Smak 1993, Cannizzo 1993, Mineshige \& Kusunose 1993). Since our ADAFs are significantly hotter than the hottest state of cataclysmic variables it seems reasonable to choose a value of $\alpha$ which is correspondingly larger. Secondly, ADAF models provide a natural explanation (Narayan 1996b) for some spectral states of black hole X-ray binaries, especially the "low state," but the models work only if $\alpha$ has a fairly large value. For the self-similar models considered by Narayan (1996b), the models required $\alpha \rightarrow 1$. With the more realistic global solutions that are now being developed (Narayan et al. 1997, Chen et al. 1997, Nakamura et al. 1996, Abramowicz et al. 1996) and that are the basis of the present paper, the requirement is a little less stringent: $\alpha \sim 0.3$ perhaps. We therefore select $\alpha=0.3$ for our baseline model. 
The parameters $\beta$ and $\delta$ are much more open, since there are very few observational constraints on their values. Fortunately, the value of $\beta$ seems to have relatively little effect on the results. Here we try two values: $\beta=0.5,0.95$. In the case of $\delta$, our standard choice is $\delta=10^{-3}\left(\sim m_{e} / m_{p}\right)$.

Figure 3a shows two models of V404 Cyg corresponding to $\beta=0.5$ and 0.95 , and standard values for the other parameters. Superimposed on the model spectra are the error box from the ASCA observations (described in §3.1) and three dots corresponding to the optical data and an arrow corresponding to the EUV upper limit (see §3.2). Table 2 lists the parameters we have chosen for these two models (as well as other models described below), and Table 3 gives a few key results of the models. The spectra shown in Figure 3a correspond to Model 1 and Model 2 in the Tables. Model 1 is our "standard model."

Interestingly, the value of $\dot{M}$ which we derive by fitting the X-ray flux is $\sim$ few $\times 10^{-10}-10^{-9} M_{\odot} \mathrm{yr}^{-1}$ for the various models presented in this paper. This is slightly smaller than, but comparable to the estimate of $\dot{M}$ obtained by King (1993) from evolutionary considerations. Thus, in our model, a reasonable fraction of the mass that is transfered by the secondary star is immediately accreted via the inner ADAF. However, some fraction of the mass does appear to be stored in the outer disk and it is this storage which presumably causes V404 Cyg to go occasionally into outburst (Mineshige \& Wheeler 1989).

Both models in Figure 3a have hard spectra in the ASCA band with photon indices $\alpha_{N} \sim 2-2.2$. These spectra are in excellent agreement with the observed spectral constraints, thus impressively confirming a key prediction of the NMY model. Note that the calculated spectra shown here are slightly softer than those obtained by NMY. The difference is a result of the more careful modeling done here, especially the use of a global flow instead of the self-similar solution employed earlier, and a better treatment of Comptonization.

Figure $3 \mathrm{~b}$ shows two thin disk models (with no ADAF or corona) which have been adjusted to fit the optical flux of V404 Cyg. The solid line corresponds to a model in which the accretion rate in the disk $\dot{M}_{d}$ is assumed to be constant as a function of radius. The fit to the data gives $\dot{M}_{d}=8 \times 10^{-10} M_{\odot} \mathrm{yr}^{-1}$. Note the huge flux that this model predicts in EUV and soft X-rays and the lack of emission in harder X-rays. Both features are inconsistent with the observations. The dashed line in Figure $3 \mathrm{~b}$ corresponds to another model in which the mass accretion rate is assumed to vary as $\dot{M}_{d}=\dot{M}_{\text {out }}\left(r / r_{\text {tr }}\right)^{3}$. This model has a nearly constant effective temperature as a function of radius and is more in line with what is expected for a quiescent thin disk which is on the cool branch of the 
standard S-curve (cf. Mineshige \& Wheeler 1989). In this case, a fit to the optical flux is obtained with $\dot{M}_{\text {out }}=5 \times 10^{-8} M_{\odot} \mathrm{yr}^{-1}$. This model does not produce any radiation in bands harder than the optical and is clearly inconsistent with the ASCA observations. Thus we conclude that, just as in A0620-00 (NMY), the data on V404 Cyg cannot be fit with any model that is composed purely of a thin accretion disk.

In the models shown in Figure 3a, nearly all the flux is from the ADAF (see Table 2). The peak in the optical/UV region of the spectrum is due to synchrotron emission, the bump in the spectrum at EUV wavelengths is due primarily to singly Compton-scattered photons, and the emission in the ASCA band is mostly doubly Compton-scattered radiation, along with some bremsstrahlung emission. (The bulk of the bremsstrahlung is at yet higher photon energies $\sim 100 \mathrm{keV}$.) The outer thin disk produces very little radiation in these models. This is because we have used a very large value for the transition radius $r_{\text {tr }}$ (selected on the basis of the $\mathrm{H} \alpha$ line width). Consequently, even though the thin disk is a much more efficient radiator than the ADAF, the fact that its energy budget is so much smaller (by a factor $\sim r_{\mathrm{tr}}^{-1}=10^{-4.4}$ ) means that its emission is quite negligible. A small amount $(\sim 10 \%)$ of the flux at infrared wavelengths is in fact from the disk, but it is not visible as a separate peak. The models presented here differ in this respect from those discussed by NMY where $r_{\text {tr }}$ was smaller $\left(\sim 10^{3}\right)$ and therefore the outer disk was a more important contributor to the optical flux.

A noteworthy feature of the models shown here is that by taking standard parameter values and adjusting just one parameter, viz. the value of $\dot{M}$, we are able to fit both the $\mathrm{X}$-ray and optical fluxes, as well as the spectral shapes in these two bands. It appears that the $\beta=0.5$ model fits the optical data better than does the $\beta=0.95$ model, but we do not believe that the difference is significant at this point. As we mentioned in $\S 2$, the models still involve approximations, some of which may modify the results somewhat; the use of non-relativistic instead of relativistic physics is a case in point. In view of this, we consider both models in Figure 1a to be satisfactory.

How sensitive are the results to the choice of parameter values? The answer is: extraordinarily little.

Figure 4a shows three models where we have kept all parameters the same as for the $\beta=0.5$ model in Figure 1a, except the black hole mass, which we varied: $M=8 M_{\odot}, 12 M_{\odot}, 16 M_{\odot}$. (These correspond to Models 3, 1, and 4, respectively, in Tables 2 and 3.) All three models fit the data very well. The synchrotron peak moves to slightly higher energies with decreasing mass. This is understood by noting that the electron temperatures in the three models are essentially the same (Table 3), and so the synchrotron peak is determined primarily by the magnetic field strength $B$. As shown 
in NMY, the field strength at a given radius $r$ scales with mass as $B \propto M^{-1 / 2}$, which explains the trend seen in Figure 4a.

Figure 4b shows four models (Models 5, 1, 6, 7 in Tables 2 and 3) where the transition radius is varied: $\log r_{\mathrm{tr}}=4.9,4.4,3.9,3.4$. The radiation from the ADAF is virtually unaffected by the change in $r_{\text {tr }}$. However, as $r_{\text {tr }}$ decreases, the blackbody emission from the outer disk becomes more important and this leads to enhanced flux in the optical band. We consider all the models in Figure $4 \mathrm{~b}$ to be acceptable fits to the data.

We have also tried varying the inclination angle $i$ in the standard model, but the differences in the model spectra are so small that the spectra lie practically on top of each other. The reason for this is two-fold. First, the ADAF is nearly spherically symmetric and optically very thin. Its emission is therefore almost precisely isotropic. The flux from the outer disk does depend strongly on the inclination, varying as cos $i$. However, the total flux from the disk is so small (see Table 3) that even the strong $\cos i$ variation leads to almost no detectable effect.

For the reasons discussed above, the exact manner in which we model the outer disk is not important for the spectral fits presented here. For instance, we have modeled the run of $\dot{M}$ in the disk and the corona somewhat arbitrarily according to equation (2.1.2). However, we could have used quite different models and the results would hardly have changed. In fact, even the choice of a constant total $\dot{M}$ between the disk and the corona is unimportant. We could have made $\dot{M}$ increase outward, as expected for a quiescent disk (Mineshige \& Wheeler 1989), without affecting the spectral fit.

Figure 5a shows five models in which $\alpha$ is varied: $\alpha=0.1,0.2,0.3,0.4,0.5$. Once again we see that the changes are minimal, and all five models are consistent with the data.

Finally, Figure 5b shows the effect of varying the parameter $\delta$. Four models are shown, corresponding to $\delta=0.001,0.00316,0.01,0.0316$. Here we see that the model spectra do show modest variations. Increasing $\delta$ corresponds to increasing the direct heating of the electrons, which leads to a higher equilibrium electron temperature (Table $3)$. This pushes all peaks to higher energies. The effect is sufficiently strong that for $\delta=0.0316$, the first Compton-scattering peak moves into the ASCA band and is seen as a steep component below about $1 \mathrm{keV}$. We consider all four models to be consistent with the present data; however, using better data it may be possible to constrain the value of $\delta$. We must caution the reader once again that we need to develop a relativistic version of the code before making such detailed comparisons. 


\subsection{Re-Analysis of A0620-00 in Quiescence}

Figure 6 shows the various observational constraints on A0620-00, as given by NMY. Relative to V404 Cyg, we see that there is significantly more information in the optical and UV bands; in fact, the data show clear evidence for a downturn of the flux in the UV, which is an important constraint on the model. The EUV flux constraint is also tighter compared to V404 Cyg. The X-ray data are, however, of poorer quality since the entire signal consists of only $39 \pm 8$ net photons from a $3 \times 10^{4} \mathrm{~s}$ observation with ROSAT (McClintock et al. 1995). We rebinned the 34 PHA channels into 6 channels. Ignoring the lowest channel, we performed a $\chi^{2}$ analysis similar to the one described in Sect. 3.1 with one important difference: here we fixed the interstellar column density at $\mathrm{N}_{\mathrm{H}}=1.6 \times 10^{21}$ whereas in the case of V404 Cyg we determined $\mathrm{N}_{\mathrm{H}}$ using the ASCA data. For $D=1.0 \mathrm{kpc}$ and an energy range of $0.4-2.5 \mathrm{keV}$, we find $\alpha_{N}=3.5 \pm 0.7(1 \sigma)$ and

$L_{\mathrm{X}} \approx 5 \times 10^{30} \mathrm{ergs} \mathrm{s}^{-1}$, which is in close agreement with our earlier results obtained using maximum-likelihood fitting (NMY). Using the coarsely binned data, we computed the $2 \sigma$ $\mathrm{X}$-ray error box shown in Figures 6-7. Note that even though the column density has been fixed, the error box is much wider than it is for V404 Cyg.

The throat of the X-ray error box corresponds to: $\log (\nu)=17.383, \log \left(\nu F_{\nu}\right)=-13.789$. As before, we have adjusted $\dot{m}$ in each model so as to fit this flux. The various other parameters of A0620-00 are reasonably well-known. Two system inclinations are discussed in the literature, $i=70^{\circ}, 40^{\circ}$ (see NMY for references), with corresponding black hole masses of $M=4.4 M_{\odot}, 12 M_{\odot}$. Barret, McClintock \& Grindlay (1996) suggest an intermediate value: $i=55^{\circ}, M=6.1 M_{\odot}$, which we adopt for our standard model. The maximum velocity from the $H \alpha$ line is $2100 \mathrm{~km} \mathrm{~s}^{-1}$, which gives $\log \left(r_{\text {tr }}\right)=4.0,3.6,3.8$ for the three inclinations mentioned above. We take the distance to the source to be 1.0 kpc (NMY).

Figures 6, 7 and Tables 4, 5 give results corresponding to a series of models of A0620-00. In general we see that the models agree fairly well with the data. The spectral slope in the X-ray band is compatible with the $2 \sigma$ range allowed by the observations, especially when one considers that we fixed $\mathrm{N}_{\mathrm{H}}$ in the analysis of the data and thereby underestimated the uncertainties. The predicted flux in the optical band is higher than the observed flux by a factor of about 2 or 3 . As already discussed in $\S 3.3$, the model has several residual uncertainties and so a discrepancy of this magnitude is not unreasonable. Interestingly, all the model spectra have downturns in the UV band, in good agreement with the observations. This is quite impressive when one recalls that in each model we adjust only one parameter, viz. $\dot{m}$, and we do not use the optical/UV data at all for determining this parameter. 
We view the good agreement in the optical/UV region of the spectrum as a confirmation of the model. The optical/UV flux in the present models is almost entirely contributed by synchrotron emission from the ADAF. This is in contrast to the models presented in NMY, where the flux was primarily from the outer thin disk. Indeed, in those models, the optical region of the spectrum was fitted by adjusting the transition radius $r_{\mathrm{tr}}$. This additional degree of freedom has been eliminated in the present models.

Figure $6 \mathrm{a}$ shows the effect of varying $\beta$. For $\beta=0.5$ (our standard model), the predicted optical flux is a little high but the shape of the spectrum is in good agreement with the data; the predicted colors agree reasonably well with the observations. The $\beta=0.95$ model, on the other hand, has the correct flux but is too red. Figures $6 \mathrm{~b}, 7 \mathrm{a}$, $7 \mathrm{~b}$ show the effect of varying the black hole mass, the transition radius, and the electron heating parameter $\delta$. Larger values of $\delta$ lead to better agreement with the X-ray data, but since the X-ray error box is fairly uncertain we feel that it would be premature to draw any conclusion from this.

\section{Summary and Discussion}

The principal result of this paper is that model spectra of the SXT source V404 Cyg in quiescence are in very good agreement with observations. The model we consider is similar to that proposed by NMY and consists of an ADAF over a wide range of radius from the black hole horizon out to a transition radius $r_{\mathrm{tr}}>10^{4}$ Schwarzschild radii, and a thin accretion disk beyond the transition radius. Nearly all the observed radiation comes from the ADAF, the flux from the outer thin disk being quite negligible. By taking standard values for various parameters (§3.2, Tables 2, 3) and adjusting only the mass accretion rate $\dot{M}$, we are able to explain all the available data, namely the optical and X-ray fluxes, the spectral shapes in these two bands, and an upper limit in EUV. In particular, we confirm the prediction of NMY that the X-ray spectrum of V404 Cyg should be hard, with a photon index of order 2. Our analysis of archival ASCA data (§3.1, Figure 1, Table 1) shows that V404 Cyg has a photon index in the range 1.8 to $2.6(90 \%$ confidence limits), which is perfectly consistent with the model prediction. Moreover, the agreement between the observations and the model is extremely robust in the sense that we have tried changing all the parameters by fairly large amounts and the fit remains good (Figures 3-5). In contrast, we find that it is impossible to fit the observations with any model that is based only on a standard thin accretion disk (see Figure 3b). These results provide a strong argument in favor of the advection-dominated accretion paradigm for quiescent black hole SXTs. 
We must emphasize that the quality of the X-ray data that the present models of V404 Cyg are being tested against is much superior to the X-ray data which NMY had to work with in the case of A0620-00. For A0620-00, McClintock et al. (1995) detected a total of only $39 \pm 8$ photons and there is a question even if all the photons came from the accretion flow (some could have come from the secondary). Even assuming that the signal is entirely from accretion, one can obtain an estimate of the photon index only by assuming a value for the hydrogen column (based on radio data), and the photon index is only moderately constrained (see the error box in Figure 6). In contrast, the ASCA data we have analyzed here correspond to $\sim 1500$ photons, and the error boxes we show in Figures 3-5 represent full $2 \sigma$ limits even after fitting for $\mathrm{N}_{\mathrm{H}}$ with the same data. The contrast in the quality of data is obvious, and the present test of the model on V404 Cyg is significantly more stringent than the one presented in NMY.

The calculation techniques employed in this paper are also an improvement over those used by NMY. We use a global solution for the flow dynamics (taken from Narayan et al. 1997 and Chen et al. 1997), instead of the simpler self-similar solution employed by NMY. We allow for non-sphericity of the ADAF (Appendix A). We also allow for full coupling among all regions of the optically thin flow in computing the Comptonization (we use over 1000 rays emanating from each radial shell in calculating the scattering probabilities, see §2.3.1), instead of the radial one-dimensional calculation used in NMY.

In view of these improvements we believe that the model spectra calculated in this paper are fairly realistic. In particular, we believe the predicted spectral slope in the X-ray band is robust. As the calculations reported by Narayan (1996b) show, ADAFs have a characteristic evolution of spectral shape as a function of increasing $\dot{M}$. Given the luminosity in the X-ray band, expressed in Eddington units, there is generally an unambiguous X-ray spectral index associated with that luminosity (see also Mahadevan 1996). In the case of V404 Cyg, we know that the mass of the black hole lies in the range $10 M_{\odot}$ to $15 M_{\odot}$ (Shahbaz et al. 1994), and from this we find that the X-ray luminosity in quiescence is $\sim 10^{-6}$ times the Eddington luminosity. This immediately constrains the slope of the spectrum to be $\alpha \gtrsim 2$. It is because of the robustness of this result that our model fit remains good despite changing the various parameters of the model by large amounts (Figures 3-5).

Further improvements to the model are, of course, possible and are clearly desirable in view of the success we have had. Principal among the improvements we seek is the inclusion of relativistic effects in the flow dynamics and radiative transfer. Abramowicz et al. (1996) have already obtained consistent relativistic flow solutions in Kerr geometry. What is needed now is to couple those solutions with radiative transfer calculations which 
include the effects of gravitational redshift, Doppler boosts and ray deflections, effects which have been neglected in the present code. It would also be preferable to avoid some of the minor approximations made in the present treatment of Comptonization (see $\S 2.3$ ). Finally, one might wish to go beyond the thermal models considered here and allow for deviations of the electron distribution function from a pure Maxwellian form.

Among all the parameters of our model, the only one which produces any significant variation in the spectrum is $\delta$. This parameter specifies the fraction of the viscous heating which goes directly into electrons. The default value of $\delta$ is the ratio of electron to proton mass $\sim 10^{-3}$, but we have also tried larger values of up to a few per cent (Figures $5 \mathrm{~b}$, $7 \mathrm{~b})$. We find that as $\delta$ increases, the equilibrium electron temperature goes up and this causes the peaks in the spectrum to move to higher energies. While the current data are not yet sensitive enough to distinguish among the various models, better data may be able to constrain the value of $\delta$. This would be extremely useful since our understanding of viscous heating in magnetized plasmas is quite primitive at this point and it is hard to imagine estimating $\delta$ with any assurance from first principles. Figures 3a and $6 \mathrm{a}$ show that the spectrum also has a modest dependence on the magnetic field parameter $\beta$. In principle, future observations may be able to measure $\beta$, which is another parameter that is hard to calculate purely from theory.

The models presented in this paper differ in one important respect from those discussed by NMY. Here, the transition between the ADAF and the outer disk occurs at a large radius, $r_{\mathrm{tr}}=10^{4.4}$ in the case of V404 Cyg and $r_{\mathrm{tr}}=10^{3.8}$ in A0620-00, whereas NMY had $r_{\text {tr }} \sim 10^{3}$. Consequently, the spectra calculated in this paper are dominated entirely by the ADAF; even in the infrared, where the disk radiates most of its flux, the disk emission is only on the order of $10 \%$ of the total flux in V404 Cyg and 25\% in A0620-00. (The exceptions are Model 7 in Tables 2, 3 and Models 5 and 6 in Tables 4, 5, where the disk radiates a larger fraction of the infrared and optical luminosity, but these models are ruled out on other grounds as discussed below.) The models of NMY, on the other hand, were dominated by emission from the outer disk. The fact that the outer disk is completely unimportant to the spectral fits presented here means that the exact details of our model of the disk are not relevant. We have for simplicity assumed that the total $\dot{M}$ in the disk plus the corona is independent of $r$ and we have adopted a specific form for the relative $\dot{M}$ in the two components (eq. 2.1.2). In fact, SXTs in quiescence are expected to have $\dot{M}$ varying with $r$ over the thin disk (cf. Mineshige \& Wheeler 1989), but the error we make by ignoring this effect is very small.

In addition, the fact that $r_{\mathrm{tr}}$ is large means that the new models are also immune to a stability problem which affected the older models (Wheeler 1996). The issue here is 
the stability of the outer thin disk. (Recall that the ADAF itself is stable, Abramowicz et al. 1995, Narayan \& Yi 1995b, Kato et al. 1996.) It is well-known that thin disks with effective temperatures $T_{\text {eff }} \lesssim 10^{4} \mathrm{~K}$ have a characteristic S-shaped dependence in the $T_{\text {eff- }} \Sigma$ plane, where $\Sigma$ is the surface density. The S-curve arises because of the effect of hydrogen ionization on the opacity. Disks are stable only if they are either on the top or bottom segment of the $\mathrm{S}$, but are unstable if they are on the middle segment. The problem with the NMY models was that they had effective temperatures which spanned (as a function of radius) a range of values from $T_{\text {eff }}<10^{3} \mathrm{~K}$ on the outside to $T_{\text {eff }} \sim 10^{4}$ $\mathrm{K}$ at the transition radius. This means that some portion of the disk in those models is on the unstable middle branch (Wheeler 1996).

Since the present models have a much larger $r_{\mathrm{tr}}$, they have a lower maximum effective temperature, $\sim 1500-2000 \mathrm{~K}$ in the case of V404 Cyg (see Table 3 ) and $\sim 3000-4000$ $\mathrm{K}$ in A0620-00 (Table 5). These models are therefore safely on the lower stable branch of the S-curve at all radii, thus eliminating Wheeler's objection. In fact, the models also eliminate another problem highlighted by Wheeler. A stable thin accretion disk in its low state (lower branch of the $\mathrm{S}$ ) has its mass accretion rate decreasing rapidly with radius, roughly as $r^{3}$ (cf. the discussion in $\S 3.3$ in connection with Figure $3 b$ ). In the NMY models, the inner edge of the disk is at such a small radius that $\dot{M}$ at the inner edge, assuming that the disk is on the lower branch of the S-curve, is much smaller than the $\dot{M}$ needed to supply the ADAF. This is no longer an issue for the models presented here. At the large transition radii we are considering now, the disk easily supplies the $\dot{M}$ of the ADAF.

In this connection we should mention that $r_{\text {tr }}$ is not very well constrained by the observations. Recall that we have estimated $r_{\mathrm{tr}}$ on the basis of the $\mathrm{H} \alpha$ line width (§3.2). From this measurement we obtained the maximum velocity $v_{\max }$ of the disk material and we then employed equation (2.1.3) to calculate $r_{\text {tr }}$. However, observationally it is quite difficult to measure $v_{\max }$ and the estimate we have used should in reality be considered only a lower bound. This means that our estimate of $r_{\mathrm{tr}}$ is actually an upper bound, as far as the $\mathrm{H} \alpha$ observations are concerned. In addition, we show in Figure 4b and Figure 7a that we can vary $r_{\text {tr }}$ by a modest amount without affecting the spectral fit. Thus, the spectrum does not constrain $r_{\text {tr }}$ either, and there is no useful lower limit on $r_{t r}$ from the observations.

We might be able to use theoretical arguments, however, to derive a lower bound on $r_{\text {tr. }}$. For instance, we could turn Wheeler's argument around and insist that $r_{\text {tr }}$ must be such that the thin disk does not cross over from the stable lower branch of the S-curve to the unstable middle branch. Tables 3 and 5 give the maximum effective temperatures of 
the outer disks in the various models presented here. Consider Models 6 and 7 of V404 Cyg in Table 3, which have smaller values of $r_{\text {tr }}$ than our standard Model 1. Model 6 has a maximum effective temperature of $4090 \mathrm{~K}$, which allows the disk to lie within the lower branch of the S-curve. This model is therefore consistent. Model 7 is, however, much too hot and is clearly ruled out since a substantial part of the disk will be in the unstable branch. Thus we can say that, within the context of the parameterisation given in equation (2.1.2), $\log \left(r_{\text {tr }}\right)$ in V404 Cyg must lie in the range $\sim 4-4.5$. (Other parameterisations will give different lower limits.) In the case of A0620-00, Table 5 shows that $\log \left(r_{\text {tr }}\right)=3.8$ is acceptable, but $\log \left(r_{\text {tr }}\right)=3.4$ and 3.0 have too high an effective temperature in the outer disk to be stable.

Another theoretical constraint comes from the requirement that the outer disk should be able to undergo a limit cycle instability, since this is believed to be the mechanism that produces the observed outbursts of SXTs (Mineshige \& Wheeler 1989). If $r_{t r}$ is too large, the outer disk may become permanently stable and there would be no limit cycle. To determine the exact limit on $r_{t r}$ from this argument we require time-dependent models of the outer disk, coupled with a detailed model of the ADAF interior. Such models are yet to be constructed.

The models presented here have a very unique geometry and make specific predictions which could be tested with future observations. Except for the $\mathrm{H} \alpha$ line emission and a small fraction of the infrared flux, virtually all the observed radiation comes from within a few tens of Schwarzschild radii of the black hole, i.e $R \lesssim 10^{8} \mathrm{~cm}$. If an eclipsing SXT is discovered (which would appear to be just a matter of time considering the rate at which new SXTs are being found), this prediction can be easily tested. Direct confirmation that the optical and UV flux come from a compact volume around the black hole would be strong verification of the model since it is difficult to imagine any competing model having this feature.

In addition, since the optical emission is from an ultra-hot ADAF, the optical flux could exhibit fairly interesting variability. It is not possible to predict the variability amplitude at this time, but the time scale can be easily estimated. For an ADAF with a large value of $\alpha \sim 1$ (as in our models), the dynamical, thermal and viscous time scales are all nearly equal: $t \sim 2 \pi\left(G M / R^{3}\right)^{-1 / 2}=0.03\left(M / 10 M_{\odot}\right)(r / 10)^{3 / 2}$ s. We thus expect quite rapid variations from the hot gas at $r \lesssim 100$. Detection of this variability would be a strong confirmation of the model since it is inconceivable that any thin disk model would produce such rapid changes. In addition, we may also expect some slower variations associated with the transition radius, since this is where the mass supply to the ADAF originates; the dynamical time scale at $r_{\mathrm{tr}}$ is an hour in the case of V404 Cyg and a few 
minutes for A0620-00.

Yet another possibility is that the optical synchrotron radiation may be polarized. The model assumes for simplicity that the magnetic field in the ADAF zone is isotropically tangled, but if the field has any residual anisotropy it might lead to polarized emission. Note, however, that the synchrotron emission is highly self-absorbed; it is not clear how much polarization is expected under these circumstances.

Quiescent SXTs and other accreting black holes with anomalously low luminosities may be among the best systems available for "proving" the existence of event horizons in black holes (NMY). Recall that the bulk of the energy in an ADAF is retained as thermal energy of the gas and is advected into the central star. This is especially obvious for the models presented in this paper, where less than $0.1 \%$ of the rest mass energy of the accreting gas is radiated (see the last column of Table 3). If the central star is a black hole with a true horizon, the advected energy disappears completely and does not contribute in any way to the observed spectrum. On the other hand, if the star is not a black hole, but a normal star with a surface, then the accreted thermal energy would ultimately be reradiated from the surface and would in fact dominate the spectrum. The successful application of the ADAF model to A0620-00 was used by NMY as an argument in favor of the black hole nature of that source. The fact that a similar model is now found to work even more impressively in V404 Cyg strengthens the argument considerably.

What can be done to make this "proof" of black hole horizons more compelling? We feel that work needs to be done on several fronts:

1. The key feature of both A0620-00 and V404 Cyg in quiescence is that they are systems with low $\dot{M}$. This is consistent with the theoretical result that optically thin ADAFs occur only at low $\dot{M}$ (Abramowicz et al. 1995, Narayan \& Yi 1995b). Theory also shows that ADAFs become progressively more advection-dominated with decreasing $\dot{M}$ (see Figure 11 in Narayan \& Yi 1995b). We thus expect the most massive advection, and therefore the strongest evidence for the disappearance of thermal energy, at the lowest mass accretion rates. Substantial progress has already been made to test this prediction. The ADAF model has been successfully applied to the low-luminosity Galactic Center source Sgr A* (Narayan, Yi \& Mahadevan 1995), to the low-luminosity nucleus of the liner galaxy NGC 4258 (Lasota et al. 1996a), and to a more general class of low-luminosity galactic nuclei (Fabian \& Rees 1995). Independent estimates of $\dot{M}$ for several of these sources strongly suggest that their luminosities are far below what is expected for accretion via a standard thin disk, which converts the usual $10 \%$ of the rest mass of the accreting material into radiation. In other words, there is clear circumstantial evidence that all these systems advect large amounts of energy into their central stars without any re-radiation. In view 
of the success of the ADAF paradigm in low-luminosity black holes, perhaps it is time to switch the argument around and ask: Is there any counter-example to the ADAF paradigm among black hole candidates which accrete at a low rate: $\dot{M}<10^{-3}-10^{-2}$ in Eddington units? In other words, is there any low $\dot{M}$ black hole which can be shown not to have an ADAF?

2. It is necessary to extend the models to higher mass accretion rates. What happens as $\dot{M}$ increases? Up to what value of $\dot{M}$ do ADAF solutions survive, and what do the brighter ADAF systems look like? How do accretion flows switch from an ADAF to a standard thin accretion disk when $\dot{M}$ becomes too large? Some preliminary answers to these questions were presented by Narayan (1996b), who explained the "low state" of black hole X-ray binaries in terms of advection-dominated accretion; more detailed work on the outburst of Nova Muscae 1991 is reported by Narayan \& McClintock (1997). Higher $\dot{M}$ systems are much brighter and easier to observe, and there is a large database on such systems, both among X-ray binaries and active galactic nuclei. There is thus enormous scope for testing the ADAF paradigm in these systems. In this connection, Yi (1996) has applied ADAF models to try and explain quasar evolution.

3. Thirdly, we feel that it is critical to demonstrate that there is a clear difference between ADAF accretion on to a black hole and ADAF accretion on to a normal star. As already mentioned, a star with a surface will re-radiate the accreted thermal energy and will therefore be significantly more luminous than a similar black hole system. The spectra too will presumably differ. There are several opportunities for exploring these differences. Many cataclysmic variables with low $\dot{M}$ appear to resemble SXTs in the sense of having a truncated outer thin disk and a central ADAF (e.g. Meyer \& Meyer-Hofmeister 1994). The interaction between the ADAF and the central white dwarf in these binaries is a topic which has hardly been explored. An even better opportunity is presented by SXTs such as Cen X-4 and Aql X-1 which are similar to black hole SXTs in many respects, but are known to consist of accreting neutron stars by the fact that they have Type $1 \mathrm{X}$-ray bursts. Indeed, as NMY pointed out, Cen X-4 in quiescence is nearly a hundred times brighter than A0620-00 (a black hole system) in quiescence; this is consistent with the ADAF paradigm. A satisfactory understanding of the luminosities and spectra of neutron star SXTs, and how these differ from the corresponding characteristics of black hole SXTs, would go a long way toward a definitive "proof" of black hole horizons. Tanaka \& Shibazaki (1996) emphasize some difficulties in reconciling ADAF models with the observed spectra of neutron star SXTs.

4. Finally, it is possible that an accreting star could get rid of the thermal energy in the accretion flow, not by swallowing it through an event horizon, but by ejecting it 
via an outflow or jet. To eliminate this loophole it is necessary to show that black hole candidates with ADAFs do not have sufficiently strong energy outflow via jets. We cannot at present judge how feasible this will be.

Acknowledgements: We thank G. Rybicki and J. Poutanen for helpful discussions on Comptonization, K. Ebisawa, Y. Tanaka and K. Terada for advice on the analysis of ASCA data, J. Casares for providing an optical spectrum of V404 Cyg, M. Garcia and I. Yi for comments on the manuscript, and an anonymous referee for useful suggestions. This research has made use of data obtained through the HEASARC Online Service, provided by the NASA/Goddard Space Flight Center. R.N. was supported in part by NASA grant NAG 5-2837. Partial support for J.E.M. was provided by the Smithsonian Institution Scholarly Studies Program. 


\section{References}

Abramowicz, M. A., Chen, X., Grantham, Lasota, J.-P. 1996, ApJ, in press (astro-ph/9607021)

Abramowicz, M. A., Chen, X., Kato, S., Lasota, J.-P, \& Regev, O. 1995, ApJ, 438, L37

Abramowicz, M. A., Czerny, B., Lasota, J.-P, \& Suszkiewicz, E. 1988, ApJ, 332, 646

Abramowicz, M. A., Jaroszyński, M., \& Sikora, M. 1978, A\&A, 63, 221

Barret, D., McClintock, J. E., Grindlay, J. E. 1996, ApJ, 473, 963

Begelman, M. C. 1978, MNRAS, 184, 53

Bjornsson, G., Abramowicz, M. A., Chen, X., \& Lasota, J.-P. 1996, ApJ, 467, 99

Blandford, R. D., \& Znajek, R. 1977, MNRAS, 179, 433

Cannizzo, J. K. 1993, in Accretion Disks in Compact Stellar Systems, ed. J. C. Wheeler (Singapore: World Scientific), 6

Cardelli, J. A., Clayton, C. C., \& Mathis, J. S. 1989, ApJ, 345, 245

Casares, J., Charles, P. A., Naylor, T., \& Pavlenko, E. P. 1993, MNRAS, 265, 834

Chen, X. 1995, MNRAS, 275, 641

Chen, X., Abramowicz, M. A., \& Lasota, J.-P. 1997, ApJ, in press astro-ph/9607020

Chen, X., Abramowicz, M. A., Lasota, J.-P., Narayan, R., \& Yi, I. 1995, ApJ, 443, L61

Coppi, P. S. \& Blandford, R. D. 1990, ApJ, 245, 453

Day et al. 1995, "The ABC Guide to ASCA Data Reduction"

Esin, A. A. 1996, ApJ, submitted

Fabian, A. C. \& Rees, M. J. 1995, MNRAS, 277, L5

Fishbone, L. G., \& Moncrief, V. 1976, ApJ, 207, 962

Frank, J., King, A., \& Raine, D. 1992, Accretion Power in Astrophysics (Cambridge: Cambridge Univ. Press)

Hawley, J. 1996, in Physics of Accretion Disks, eds. S. Kato, S. Inagaki, S. Mineshige, J. Fukue (Gordon and Breach)

Honma, F. 1996, PASJ, 48, 77

Jones, F. C. 1968, ApJ, 167, 1159

Kato, S., Abramowicz, M. A., \& Chen, X. 1996, PASJ, 48, 67

Katz, J. 1977, ApJ, 215, 265

King, A. R. 1993, MNRAS, 260, L5

Kusunose, M., \& Mineshige, S. 1996, ApJ, 468, 330

Lasota, J.-P., Abramowicz, M. A., Chen, X., Krolik, J., Narayan, R., \& Yi, I. 1996a, ApJ, 462, 142

Lasota, J.-P., Narayan, R., \& Yi, I. 1996b, A\&A, in press (astro-ph/9605011)

Mahadevan, R. 1996, ApJ, in press (astro-ph/9609107)

Mahadevan, R., Narayan, R., \& Yi, I. 1996, ApJ, 465, 327

Marsh, T. R., Robinson, E. L., \& Wood, J. H. 1994, MNRAS, 266, 137

McClintock, J. E., Horne, K., \& Remillard, R. A. 1995, ApJ, 442, 358

Meyer, F., \& Meyer-Hofmeister, E. 1994, A\&A, 288, 175

Mihalas, 1978, Stellar Atmospheres (San Francisco: Freeman)

Mineshige, S. \& Kusunose, M. 1993, in Accretion Disks in Compact Stellar Systems, ed. J. C. Wheeler (Singapore: World Scientific), 370

Mineshige, S., \& Wheeler, J. C. 1989, ApJ, 343, 241

Nakamura, K., Matsumoto, R., Kusunose, M., \& Kato, S. 1996, PASJ, 48 (Oct. 20)

Narayan, R. 1996a, in Physics of Accretion Disks, eds. S. Kato, S. Inagaki, S. Mineshige, J. Fukue (Gordon and Breach), 15

Narayan, R. 1996b, ApJ, 462, 136 
Narayan, R. 1997, Proc. IAU Colloq. No 163, Accretion Phenomena \& Related Outflows, A.S.P. Conf. Series., ed. Wickramasinghe, D.T., Ferrario, L. \& Bicknell, G.V.

Narayan, R., Kato, S., \& Honma, F. 1997, ApJ, in press (astro-ph/9607019)

Narayan, R., \& McClintock, J. E. 1997, ApJ, in preparation

Narayan, R., McClintock, J. E., \& Yi, I. 1996, ApJ, 457, 821 (NMY)

Narayan, R., \& Yi, I. 1994, ApJ, 428, L13

Narayan, R., \& Yi, I. 1995a, ApJ, 444, 231

Narayan, R., \& Yi, I. 1995b, ApJ, 452, 710

Narayan, R., Yi, I., \& Mahadevan, R. 1995, Nature, 374, 623

Novikov, I. D., \& Thorne, K. S. 1973, in Blackholes ed. C. DeWitt \& B. DeWitt (New York: Gordon \& Breach), 345

Pacholczyk, A. G. 1970, Radio Astrophysics (San Francisco: Freeman)

Paczyński, B., \& Wiita, P. J. 1980, A\&A, 88, 23

Petrosian, V. 1981, ApJ, 251, 727

Phinney, E. S. 1981, in Plasma Astrophysics, ed. T. D. Guyenne \& G. Levy (ESA SP-161), 337

Piran, T. 1978, ApJ, 221, 652

Poutanen, J., \& Svensson, R. 1996, ApJ, in press (astro-ph/9605073)

Rees, M. J., Begelman, M. C., Blandford, R. D., \& Phinney, E. S. 1982, Nature, 295, 17

Shahbaz, T., Ringwald, F. A., Bunn, J. C., Naylor, T., Charles, P. A., \& Casares, J. 1994 MNRAS, 271, L10

Shapiro, S. I., Lightman, A. P., \& Eardley, D. M. 1976, ApJ, 204, 187

Smak, J. 1993, Acta Astron., 43, 101

Spruit, H., Matsuda, T., Inoue, M., \& Sawada, K. 1987, MNRAS, 229, 517

Stepney, S., \& Guilbert, P. W. 1983, MNRAS, 204, 1269

Sunyaev, R. A., \& Titarchuk, L. G. 1980, A\&A, 86, 121

Svensson, R. 1982, ApJ, 258, 335

Takahara, F., \& Tsuruta, S. 1982, Prog. Theor. Phys., 67, 485

Tanaka, Y. \& Shibazaki, N. 1996, ARAA, 34, in press

van Paradijs, J., \& McClintock, J. E. 1995, in X-ray Binaries, ed. W. H. G. Lewin, J. van Paradijs, \& E. P. J. van den Heuvel (Cambridge: Cambridge Univ. Press), 58

Wagner, R. M., Kreidl, T. J., Howell, S. B., \& Starrfield, S. G. 1992, ApJ, 401, L97

Wagner, R. M., Starrfield, S. G., Hjellming, R. M., Howell, S. B., \& Kreidl, T. J. 1994, ApJ, 401, L97

Wheeler, J. C. 1996, in Relativistic Astrophysics, eds. B. Jones \& D. Markovic (Cambridge Univ. Press) astro-ph/9606119)

Yi, I. 1996, ApJ, in press (astro-ph/9609146) 


\section{A. Non-Spherical Stucture of the ADAF}

Using the results of Narayan \& Yi (1995a) as a guide, we assume that at each spherical radius $R$, the angular velocity $\Omega$ and the isothermal sound speed $c_{s}=(p / \rho)^{1 / 2}$ are independent of the polar angle $\theta$. Consider the condition for "hydrostatic equilibrium" in the $\theta$ direction. Since the gravitational force vanishes along this direction, we need consider only the pressure gradient and the centrifugal term. This gives

$$
\frac{1}{\rho} \frac{d p}{R d \theta}=\Omega^{2} R \sin \theta \cos \theta,
$$

which integrates to

$$
\rho=\rho_{0} \exp \left[-\frac{\Omega^{2} R^{2} \cos ^{2} \theta}{2 c_{s}^{2}}\right],
$$

We thus obtain the variation of density with polar angle.

Let us assume that the radial velocity $v$ is independent of $\theta$. (This is not true, but it is an acceptable approximation for the present purpose). Then the mass accretion rate corresponding to (A.2) is

$$
\dot{M}=4 \pi \sqrt{\frac{\pi}{2}} \frac{\rho_{0} v R c_{s}}{\Omega} \operatorname{erf}\left(\frac{\Omega R}{\sqrt{2} c_{s}}\right) .
$$

This relation determines $\rho_{0}$ in terms of $\dot{M}$.

The global solutions calculated by Narayan, Kato \& Honma (1997) and Chen, Abramowicz \& Lasota (1997) give $\Omega, v$ and $c_{s}^{2}$ as functions of radius $R$. By substituting these in the above formulae, we see that we can calculate the density $\rho(R, \theta)$ in the two-dimensional $(R, \theta)$ plane. Narayan (1997) shows isodensity contours of a typical solution with $\alpha=0.3$ and $\beta=0.5$.

For the spectral calculations of this paper, a density that varies with $\theta$ is inconvenient since the various emission processes vary as different powers of the density. Therefore, we simplify matters by replacing the above density profile by one in which the density is equal to $\rho_{0}$ over a certain range of angle on either side of the equator and vanishes near the two poles. Define the angle $\theta_{H}$ by

$$
\cos \theta_{H}=\sqrt{\frac{\pi}{2}} \frac{c_{s}}{\Omega R} \operatorname{erf}\left(\frac{\Omega R}{\sqrt{2} c_{s}}\right) .
$$

Substituting in eq. (A.3), we see that

$$
\dot{M}=4 \pi \rho_{0} v R^{2} \cos \theta_{H} .
$$


Thus, the density profile (A.2) may be replaced by a simpler model in which the density is constant over the range $\theta_{H}<\theta<\pi-\theta_{H}$ and vanishes over the poles. We make use of this simplified model for the spectral calculations described in the paper. 


\section{Figure Captions}

Figure 1: Combined unfolded GIS and SIS spectra of V404 Cyg for the power law model.

Figure 2: (a) Allowed grid of variations of the column density $\left(\mathrm{N}_{\mathrm{H}}\right)$ and the power law photon index $(\alpha)$ in V404 Cyg. The contours encompass the $68 \%, 95 \%$ and $99 \%$ confidence levels. (b) Allowed grid of variations of the normalization flux at $1 \mathrm{keV}$ and $\alpha$.

Figure 3: (a) Spectra corresponding to Models 1 and 2 of V404 Cyg in quiescence (see Tables 2 and 3). The models correspond to $\beta=0.5$ and 0.95 respectively, with standard values for the other parameters. The three dots represent measured optical fluxes (from NMY) and the the error box in the X-ray band, which corresponds to a $2 \sigma$ deviation, is obtained from our analysis of ASCA data (§3.1). The mass accretion rates in the two models have been adjusted so that the calculated spectra pass through the "throat" of the error box. The peak on the left in the two spectra is due to synchrotron radiation from the ADAF. The next bump arises from single Compton scattering, and the peak on the right is the result of bremsstrahlung emission. The radiation in the ASCA band is primarily from doubly and triply Compton scattered photons combined with the low-energy tail of the bremsstrahlung peak. (b) Attempts to fit the data with two pure thin disk models (see text for details). The models have been adjusted to pass through the optical data. The fit in the X-ray band is extremely poor.

Figure 4: (a) Spectra corresponding to three models of V404 Cyg in which the mass of the black hole is varied: $M=8,12,16 M_{\odot}$ (Models 3, 1, 4 in Tables 2, 3). Note that all three models fit the data very well. (b) Four models which differ in the transition radius: $\log r_{\mathrm{tr}}=4.9,4.4,3.9,3.4$ (Models 5, 1, 6, 7 in Tables 2, 3). All four models fit the observations well. However, Model 7 has a large effective temperatures in the disk and may be ruled out (see $\S 4$ ).

Figure 5: (a) Spectra of five models of V404 Cyg which differ in the choice of viscosity parameter: $\alpha=0.1,0.2,0.3,0.4,0.5$ (Models 8, 9, 1, 10, 11 in Tables 2, 3). All five models fit the data very well. (b) Four models which differ in the assumed direct heating of the electrons: $\delta=0.001,0.00316,0.01,0.0316$ (Models 1, 12, 13, 14 in Tables 2, 3). All models again fit the data satisfactorily. However, the model spectra differ from each other in the ASCA band, and could in principle be distinguished with more sensitive observations.

Figure 6: (a) Spectra of Models 1 and 2 of A0620-00 in quiescence (see Tables 4, 5). The models correspond to $\beta=0.5$ and 0.95 respectively, with standard values for the other parameters. The optical/UV fluxes and EUV limit are taken from NMY. The X-ray error box corresponds to $2 \sigma$ limits with fixed $\mathrm{N}_{\mathrm{H}}$ (see $\S 3.4$ for details). We consider both 
models to be satisfactory. (b) Three models of A0620-00 in which the black hole mass is varied: $M=4.4 M_{\odot}, 6.1 M_{\odot}, 12 M_{\odot}($ Models $3,1,4$ in Tables 4,5$)$.

Figure 7: (a) Three models of A0620-00 in which the transition radius is varied: $\log \left(r_{\text {tr }}\right)=3.8,3.4,3.0$ (Models 1, 5, 6 in Tables 4,5). Models 5 and 6 have large effective temperatures in the outer disk and can probably be ruled out (see §4). (b) Four models of A0620-00 which differ in the assumed heating of the electrons: $\delta=0.001,0.00316,0.01,0.0316$ (Models 1, 7, 8, 9 in Tables 4, 5). As in the case of V404 Cyg (Fig. 5b), the models predict different spectral slopes in the X-ray band and could in principle be distinguished with sensitive observations. 


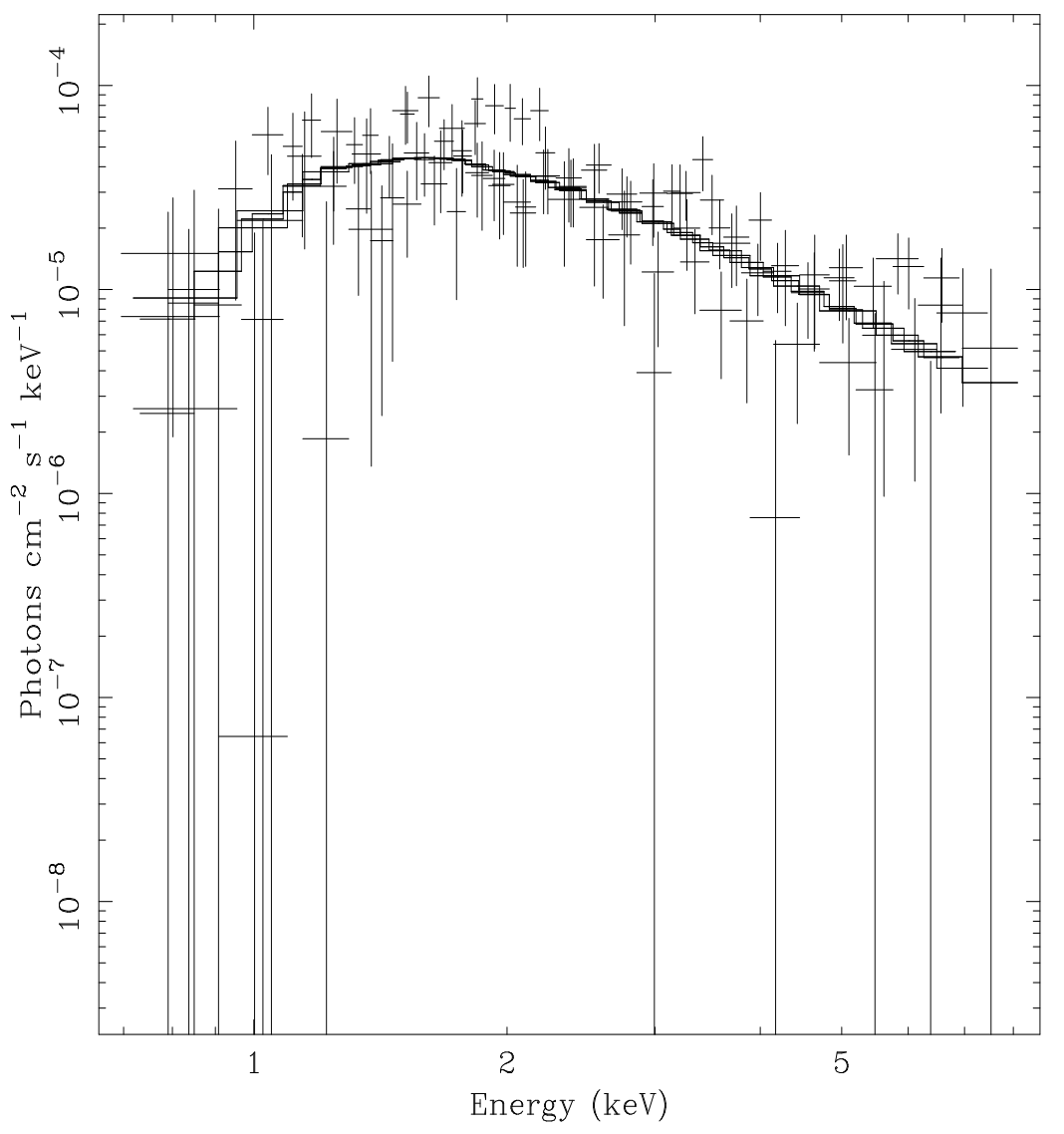

Figure 1 


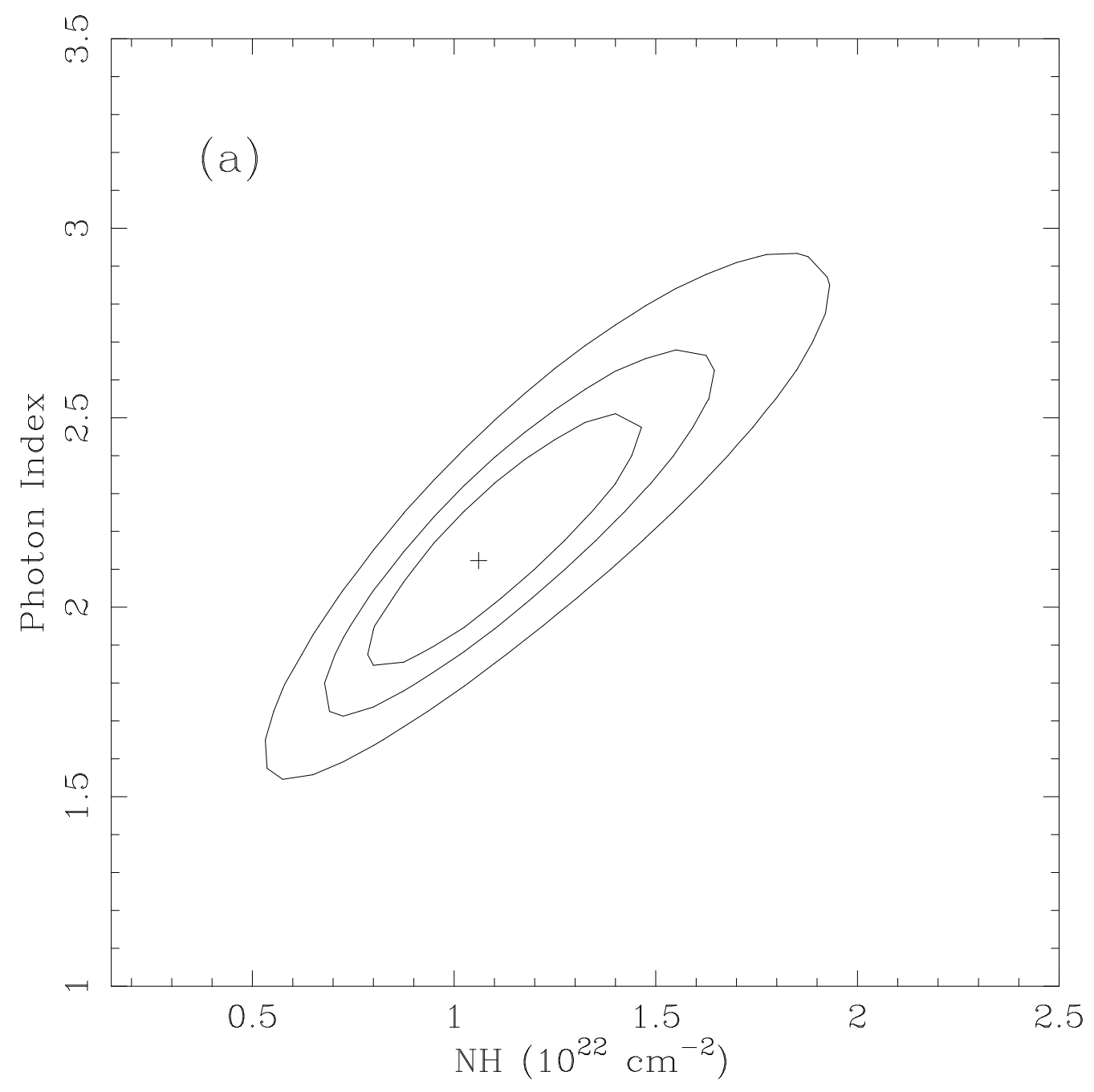

Figure 2a 


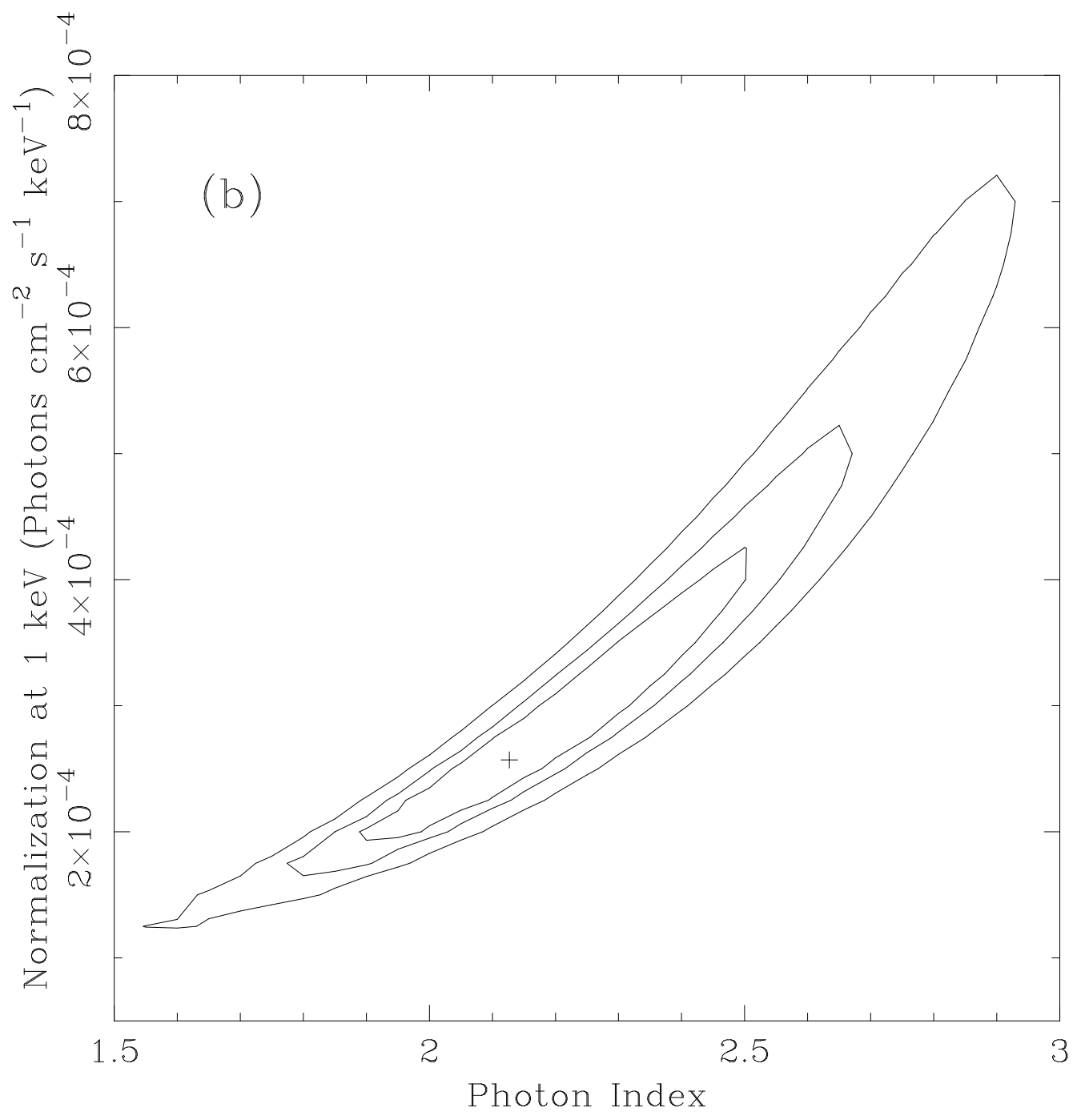

Figure 2b 

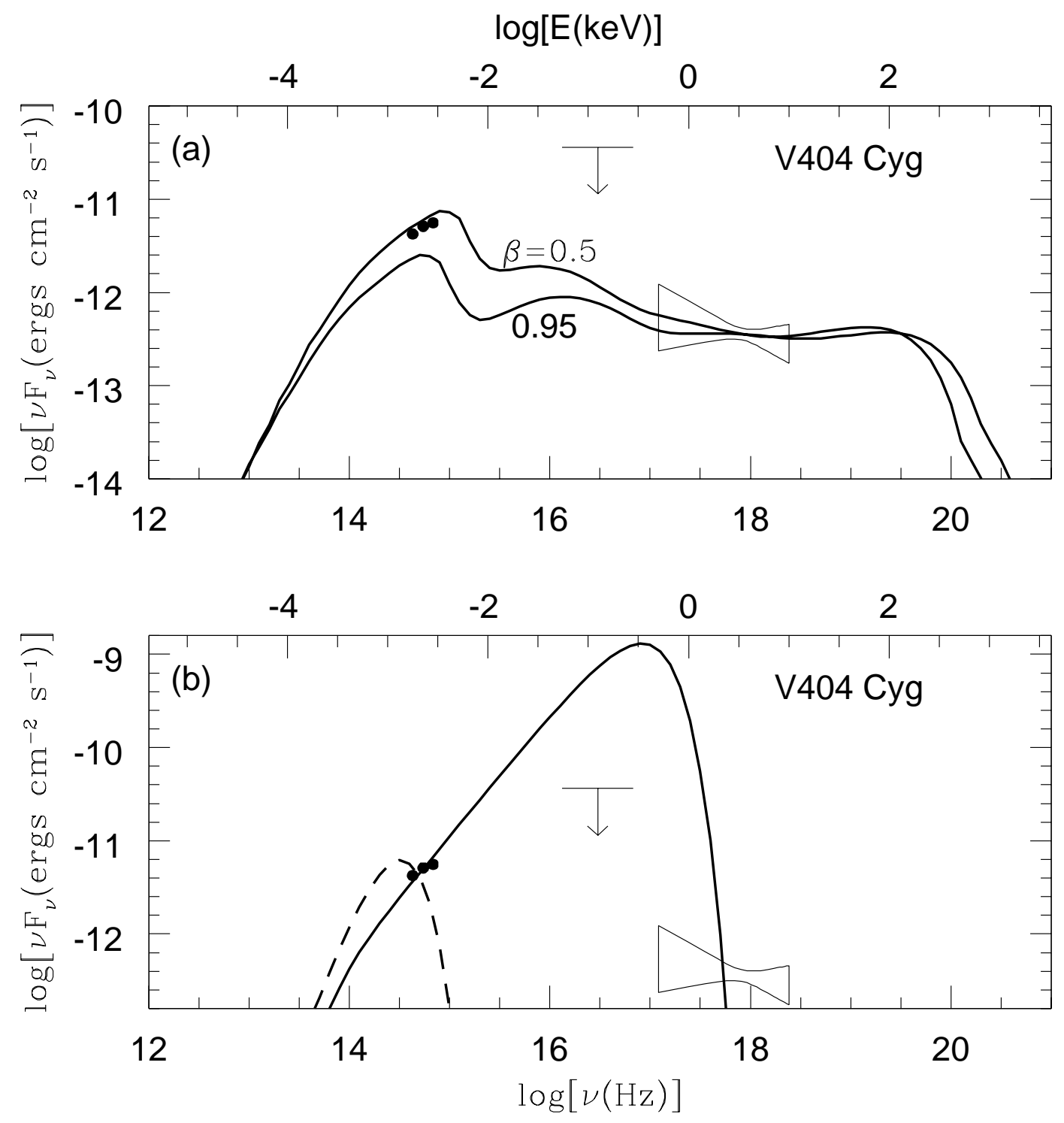

Figure 3 

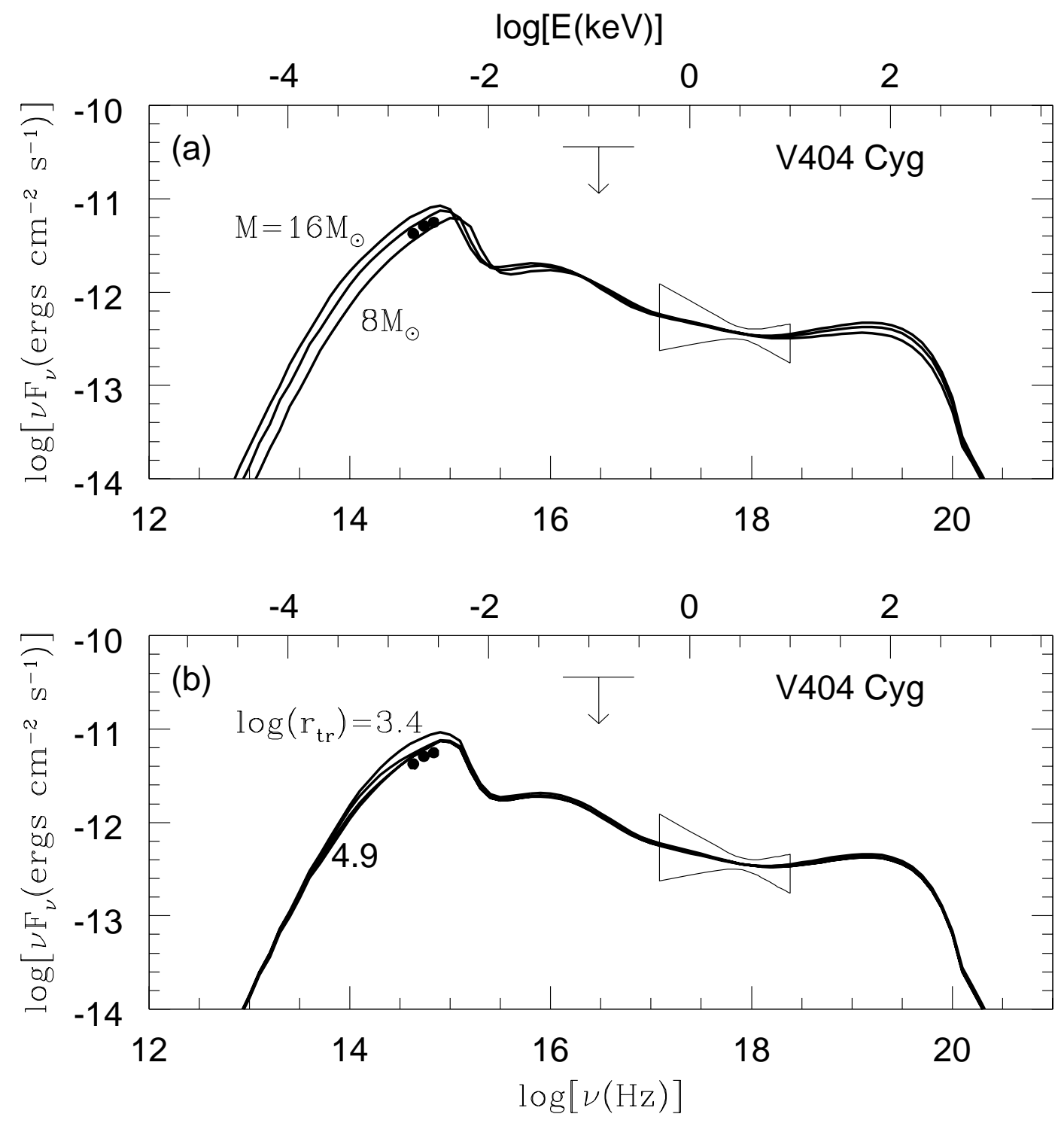

Figure 4 

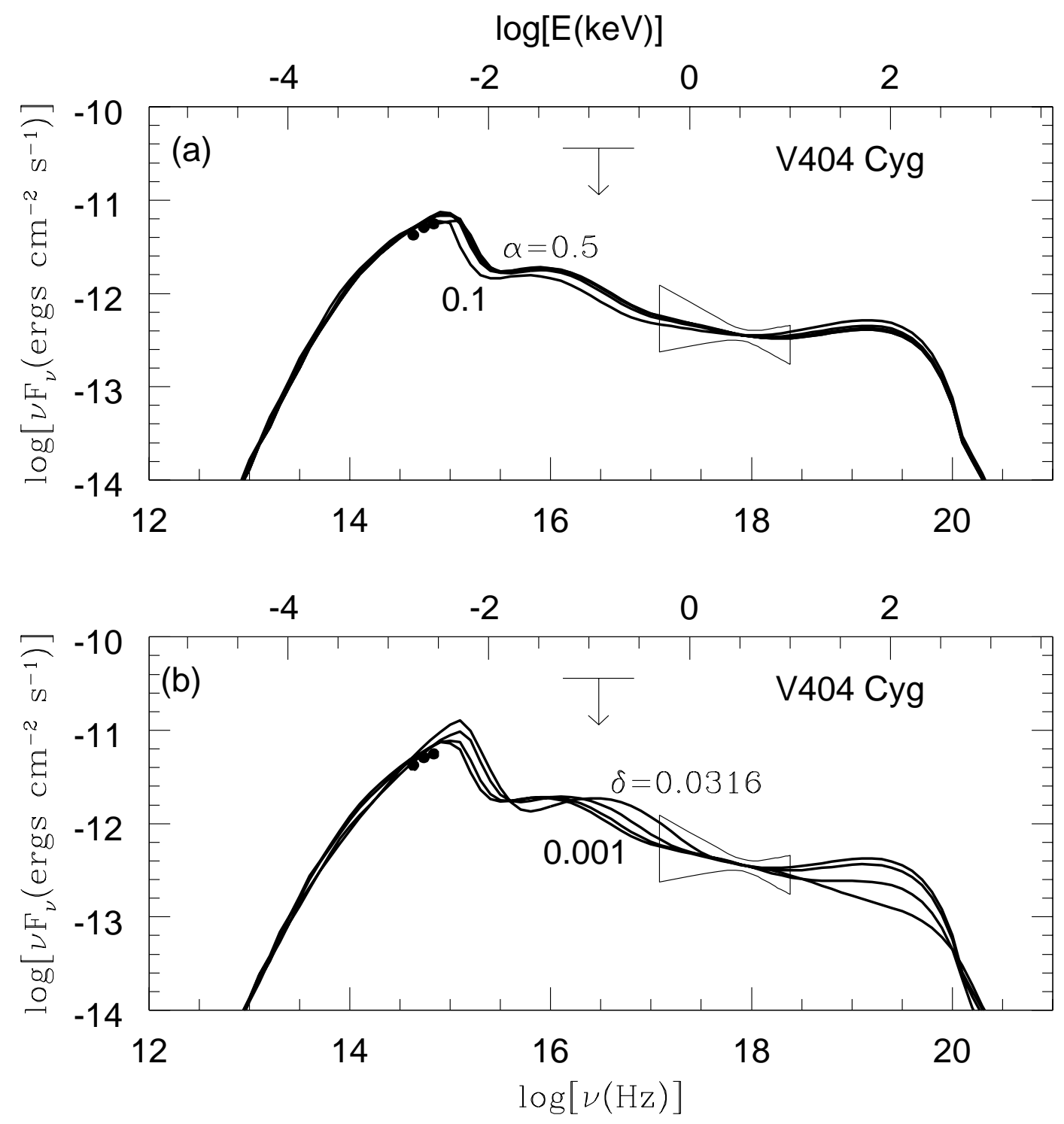

Figure 5 

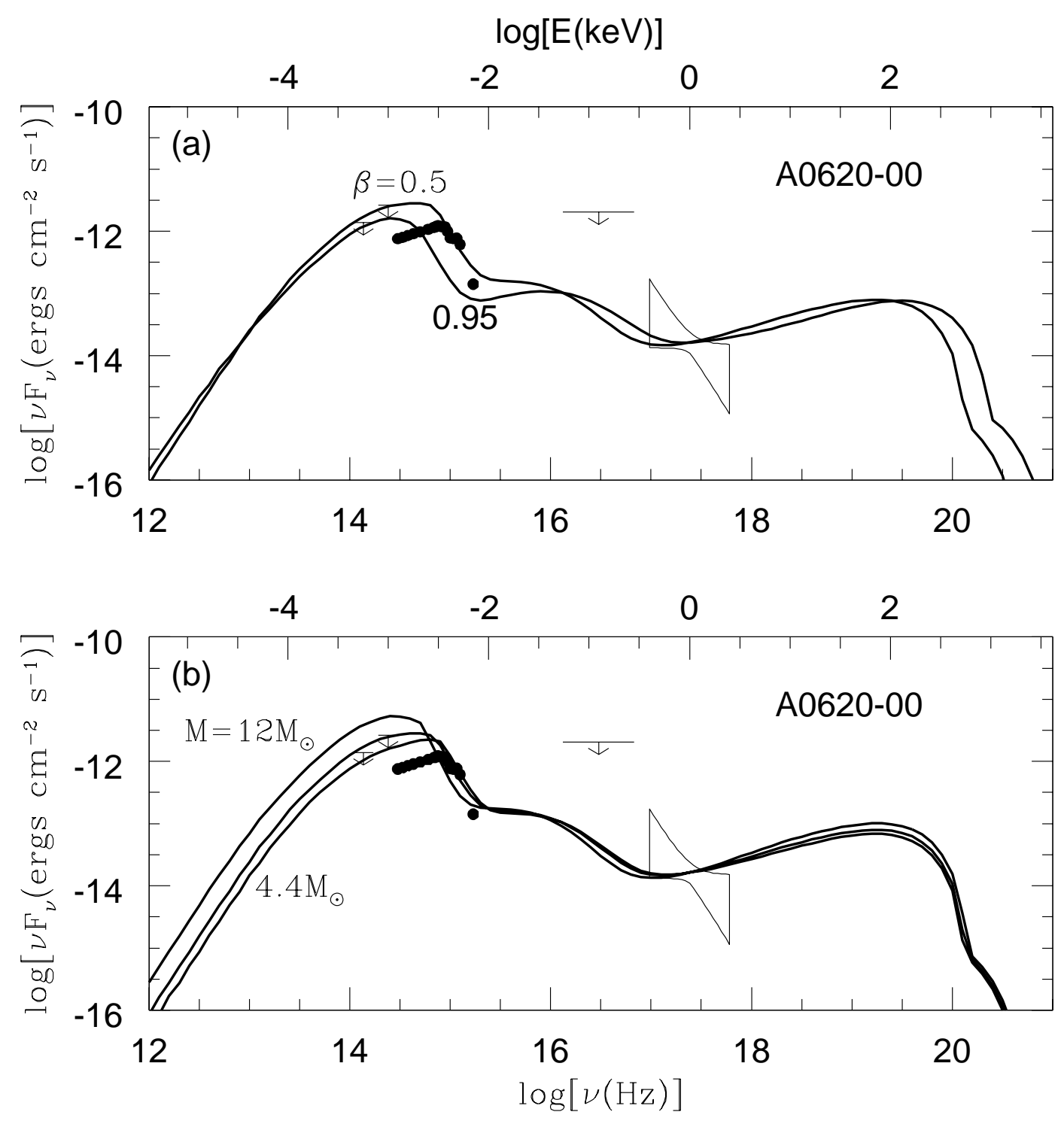

Figure 6 

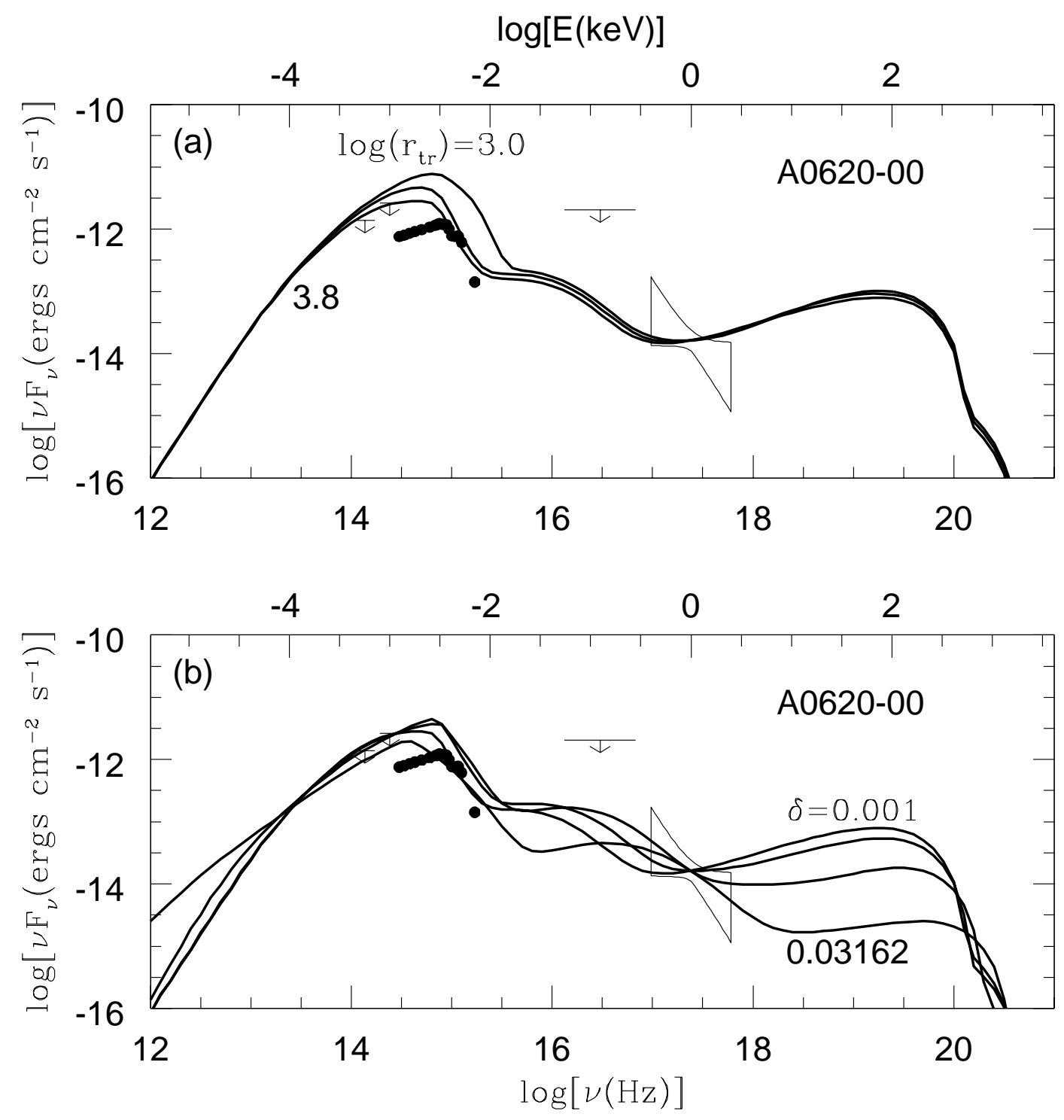

Figure 7 


\begin{tabular}{|ccccc|}
\hline Model & $\mathrm{N}_{\mathrm{H}}\left(\times 10^{22} \mathrm{~cm}^{-2}\right)$ & $\alpha, \mathrm{kT}$ & Flux $^{\mathrm{a})} @ 1 \mathrm{keV}$ & Red. $\chi^{2}$ (d.o.f) \\
\hline $\mathrm{PL}$ & $1.10_{-0.40}^{+0.30}$ & $2.10_{-0.30}^{+0.50}$ & $2.50_{-0.80}^{+2.00}$ & $0.99(105)$ \\
\hline $\mathrm{TB}$ & $0.81_{-0.40}^{+0.30}$ & $4.60_{-1.54}^{+3.60}$ & $2.12_{-0.46}^{+0.77}$ & $1.01(105)$ \\
\hline $\mathrm{BB}$ & $0.18_{-0.18}^{+0.22}$ & $0.82_{-0.08}^{+0.08}$ & $0.59_{-0.06}^{+0.06}$ & $1.14(105)$ \\
\hline \hline
\end{tabular}

Table 1: Spectral parameters of V404 Cyg as derived from the archival $40 \mathrm{ksec}$ observation. The fit is performed between 0.7 and $8.5 \mathrm{keV}$, and the uncertainties are computed at the $90 \%$ confidence level for joint variation of all parameters. Models: PL: Power Law, TB=Thermal Bremsstrahlung, $\mathrm{BB}=$ Blackbody. $\alpha$ is the photon index. $\mathrm{kT}$ is the temperature of either the TB or BB model.

a) $\times 10^{-4}$ Phot $\mathrm{cm}^{-2} \mathrm{~s}^{-1} \mathrm{keV}^{-1}$ 


\begin{tabular}{|cccccccc|}
\hline Model & $\mathrm{M}\left(M_{\odot}\right)$ & $\mathrm{i}\left(^{o}\right)$ & $\log \left(r_{\text {tr }}\right)$ & $\alpha$ & $\beta$ & $\delta$ & $\dot{M}\left(M_{\odot} \mathrm{yr}^{-1}\right)$ \\
\hline 1 & 12 & 56 & 4.4 & 0.3 & 0.5 & 0.001 & $1.22 \times 10^{-9}$ \\
2 & 12 & 56 & 4.4 & 0.3 & 0.95 & 0.001 & $1.00 \times 10^{-9}$ \\
\hline 3 & 8 & 56 & 4.4 & 0.3 & 0.5 & 0.001 & $9.12 \times 10^{-10}$ \\
4 & 16 & 56 & 4.4 & 0.3 & 0.5 & 0.001 & $1.49 \times 10^{-9}$ \\
\hline 5 & 12 & 56 & 4.9 & 0.3 & 0.5 & 0.001 & $1.22 \times 10^{-9}$ \\
6 & 12 & 56 & 3.9 & 0.3 & 0.5 & 0.001 & $1.23 \times 10^{-9}$ \\
7 & 12 & 56 & 3.4 & 0.3 & 0.5 & 0.001 & $1.26 \times 10^{-9}$ \\
\hline 8 & 12 & 56 & 4.4 & 0.1 & 0.5 & 0.001 & $5.29 \times 10^{-10}$ \\
9 & 12 & 56 & 4.4 & 0.2 & 0.5 & 0.001 & $8.99 \times 10^{-10}$ \\
10 & 12 & 56 & 4.4 & 0.4 & 0.5 & 0.001 & $1.52 \times 10^{-9}$ \\
11 & 12 & 56 & 4.4 & 0.5 & 0.5 & 0.001 & $1.84 \times 10^{-9}$ \\
\hline 12 & 12 & 56 & 4.4 & 0.3 & 0.5 & 0.00316 & $1.11 \times 10^{-9}$ \\
13 & 12 & 56 & 4.4 & 0.3 & 0.5 & 0.01 & $8.16 \times 10^{-10}$ \\
14 & 12 & 56 & 4.4 & 0.3 & 0.5 & 0.0316 & $4.06 \times 10^{-10}$ \\
\hline \hline
\end{tabular}

Table 2: Parameters of models of V404 Cyg shown in Figures 3-5. Model 1 is the baseline model, which incorporates the standard values of the parameters (see §3.2) 


\begin{tabular}{|cccccc|}
\hline Model & $L_{\text {ADAF }}\left(\mathrm{ergs} \mathrm{s}^{-1}\right)$ & $T_{\mathrm{e}, \mathrm{ADAF}, \max }(\mathrm{K})$ & $L_{\text {disk }}\left(\mathrm{ergs} \mathrm{s}^{-1}\right)$ & $T_{\text {eff,disk,max }}(\mathrm{K})$ & $\frac{L_{\mathrm{ADAF}}+L_{\text {disk }}}{\text { Mc }}{ }^{2}$ \\
\hline 1 & $3.30 \times 10^{34}$ & $2.83 \times 10^{9}$ & $2.52 \times 10^{32}$ & 1920 & $4.81 \times 10^{-4}$ \\
2 & $1.48 \times 10^{34}$ & $4.84 \times 10^{9}$ & $1.75 \times 10^{32}$ & 1680 & $2.63 \times 10^{-4}$ \\
\hline 3 & $2.82 \times 10^{34}$ & $2.77 \times 10^{9}$ & $1.99 \times 10^{32}$ & 2270 & $5.49 \times 10^{-4}$ \\
4 & $3.65 \times 10^{34}$ & $2.88 \times 10^{9}$ & $2.98 \times 10^{32}$ & 1710 & $4.37 \times 10^{-4}$ \\
\hline 5 & $3.31 \times 10^{34}$ & $2.83 \times 10^{9}$ & $1.80 \times 10^{31}$ & 1020 & $4.80 \times 10^{-4}$ \\
6 & $3.35 \times 10^{34}$ & $2.82 \times 10^{9}$ & $1.26 \times 10^{33}$ & 4090 & $4.99 \times 10^{-4}$ \\
7 & $3.48 \times 10^{34}$ & $2.82 \times 10^{9}$ & $4.80 \times 10^{33}$ & 9490 & $5.55 \times 10^{-4}$ \\
\hline 8 & $2.90 \times 10^{34}$ & $2.85 \times 10^{9}$ & $1.86 \times 10^{32}$ & 2050 & $9.72 \times 10^{-4}$ \\
9 & $3.21 \times 10^{34}$ & $2.82 \times 10^{9}$ & $2.22 \times 10^{32}$ & 1930 & $6.34 \times 10^{-4}$ \\
10 & $3.26 \times 10^{34}$ & $2.83 \times 10^{9}$ & $2.88 \times 10^{32}$ & 1900 & $3.83 \times 10^{-4}$ \\
11 & $3.11 \times 10^{34}$ & $2.85 \times 10^{9}$ & $3.29 \times 10^{32}$ & 1950 & $3.01 \times 10^{-4}$ \\
\hline 12 & $3.39 \times 10^{34}$ & $2.89 \times 10^{9}$ & $2.26 \times 10^{32}$ & 1860 & $5.44 \times 10^{-4}$ \\
13 & $3.64 \times 10^{34}$ & $3.75 \times 10^{9}$ & $1.60 \times 10^{32}$ & 1670 & $7.90 \times 10^{-4}$ \\
14 & $4.19 \times 10^{34}$ & $5.63 \times 10^{9}$ & $7.62 \times 10^{31}$ & 1380 & $1.83 \times 10^{-3}$ \\
\hline \hline
\end{tabular}

Table 3: Luminosities and temperatures of the ADAF and thin disk, corresponding to the models of V404 Cyg listed in Table 2. $L_{\mathrm{ADAF}}$ is the total luminosity escaping from the ADAF and corona, integrated over all directions. $T_{\mathrm{e}, \mathrm{ADAF} \text { max }}$ is the maximum electron temperature in the ADAF. $L_{\text {disk }}$ is the luminosity escaping from the outer disk. $T_{\text {eff,max }}$ is the maximum effective temperature in the disk. The last column gives the overall efficiency of the accretion flow.

\begin{tabular}{|cccccccc|}
\hline Model & $\mathrm{M}\left(M_{\odot}\right)$ & $\mathrm{i}\left({ }^{o}\right)$ & $\log \left(r_{\text {tr }}\right)$ & $\alpha$ & $\beta$ & $\delta$ & $M\left(M_{\odot} \mathrm{yr}^{-1}\right)$ \\
\hline 1 & 6.1 & 55 & 3.8 & 0.3 & 0.5 & 0.001 & $1.31 \times 10^{-10}$ \\
2 & 6.1 & 55 & 3.8 & 0.3 & 0.95 & 0.001 & $1.20 \times 10^{-10}$ \\
\hline 3 & 4.4 & 70 & 4.0 & 0.3 & 0.5 & 0.001 & $1.45 \times 10^{-10}$ \\
4 & 12 & 40 & 3.6 & 0.3 & 0.5 & 0.001 & $1.04 \times 10^{-10}$ \\
\hline 5 & 6.1 & 55 & 3.4 & 0.3 & 0.5 & 0.001 & $1.41 \times 10^{-10}$ \\
6 & 6.1 & 55 & 3.0 & 0.3 & 0.5 & 0.001 & $1.49 \times 10^{-10}$ \\
\hline 7 & 6.1 & 55 & 3.8 & 0.3 & 0.5 & 0.00316 & $1.03 \times 10^{-10}$ \\
8 & 6.1 & 55 & 3.8 & 0.3 & 0.5 & 0.01 & $4.57 \times 10^{-11}$ \\
9 & 6.1 & 55 & 3.8 & 0.3 & 0.5 & 0.0316 & $7.46 \times 10^{-12}$ \\
\hline \hline
\end{tabular}

Table 4: Parameters of models of A0620-00 shown in Figures 6 and 7. 


\begin{tabular}{|cccccc|}
\hline Model & $L_{\mathrm{ADAF}}\left(\mathrm{ergs}^{-1}\right)$ & $T_{\mathrm{e}, \mathrm{ADAF}, \max }(\mathrm{K})$ & $L_{\text {disk }}\left(\mathrm{ergs} \mathrm{s}^{-1}\right)$ & $T_{\text {eff,disk,max }}(\mathrm{K})$ & $\frac{L_{\mathrm{ADAF}}+L_{\text {disk }}}{\mathrm{Mc}^{2}}$ \\
\hline 1 & $1.04 \times 10^{33}$ & $2.78 \times 10^{9}$ & $1.54 \times 10^{32}$ & 3710 & $1.60 \times 10^{-4}$ \\
2 & $5.06 \times 10^{32}$ & $5.04 \times 10^{9}$ & $1.40 \times 10^{32}$ & 3620 & $9.51 \times 10^{-5}$ \\
\hline 3 & $8.97 \times 10^{32}$ & $2.69 \times 10^{9}$ & $6.51 \times 10^{31}$ & 2930 & $1.17 \times 10^{-4}$ \\
4 & $1.36 \times 10^{33}$ & $2.93 \times 10^{9}$ & $4.22 \times 10^{32}$ & 4160 & $3.03 \times 10^{-4}$ \\
\hline 5 & $1.17 \times 10^{33}$ & $2.75 \times 10^{9}$ & $4.98 \times 10^{32}$ & 7520 & $2.08 \times 10^{-4}$ \\
6 & $1.27 \times 10^{33}$ & $2.73 \times 10^{9}$ & $1.42 \times 10^{33}$ & 15200 & $3.18 \times 10^{-4}$ \\
\hline 7 & $1.33 \times 10^{33}$ & $3.55 \times 10^{9}$ & $1.21 \times 10^{32}$ & 3490 & $2.49 \times 10^{-4}$ \\
8 & $1.46 \times 10^{33}$ & $5.65 \times 10^{9}$ & $5.37 \times 10^{31}$ & 2850 & $5.86 \times 10^{-4}$ \\
9 & $7.40 \times 10^{32}$ & $1.24 \times 10^{10}$ & $9.11 \times 10^{30}$ & 1840 & $1.77 \times 10^{-3}$ \\
\hline \hline
\end{tabular}

Table 5: Luminosities and temperatures of the ADAF and thin disk, corresponding to the models of A0620-00 listed in Table 4. $L_{\mathrm{ADAF}}$ is the total luminosity escaping from the ADAF and corona, integrated over all directions. $T_{\mathrm{e}, \mathrm{ADAF} \text {,max }}$ is the maximum electron temperature in the ADAF. $L_{\text {disk }}$ is the luminosity escaping from the outer disk. $T_{\text {eff,max }}$ is the maximum effective temperature in the disk. The last column gives the overall efficiency of the accretion flow. 